\title{
An updated synthesis of the diazo-transfer reagent imidazole-1-sulfonyl azide hydrogen sulfate
}

\author{
Garrett T. Potter, Gordon C. Jayson, Gavin J. Miller and John M. Gardiner*
}

gardiner@manchester.ac.uk

\section{Supporting Information}

Contents:

${ }^{1} \mathrm{H} /{ }^{13} \mathrm{C} \mathrm{NMR}$

pp. S2-S5

FTIR

pp. S6-S7

MALDI

pp. S8-S12

Photographic Stepwise Procedure

pp. S13-S33

Representative Diazotransfer Protocol

p. 34 
1- $\mathrm{H}_{2} \mathbf{S O}_{4}{ }^{1} \mathrm{HNMR}$ (Method A product)

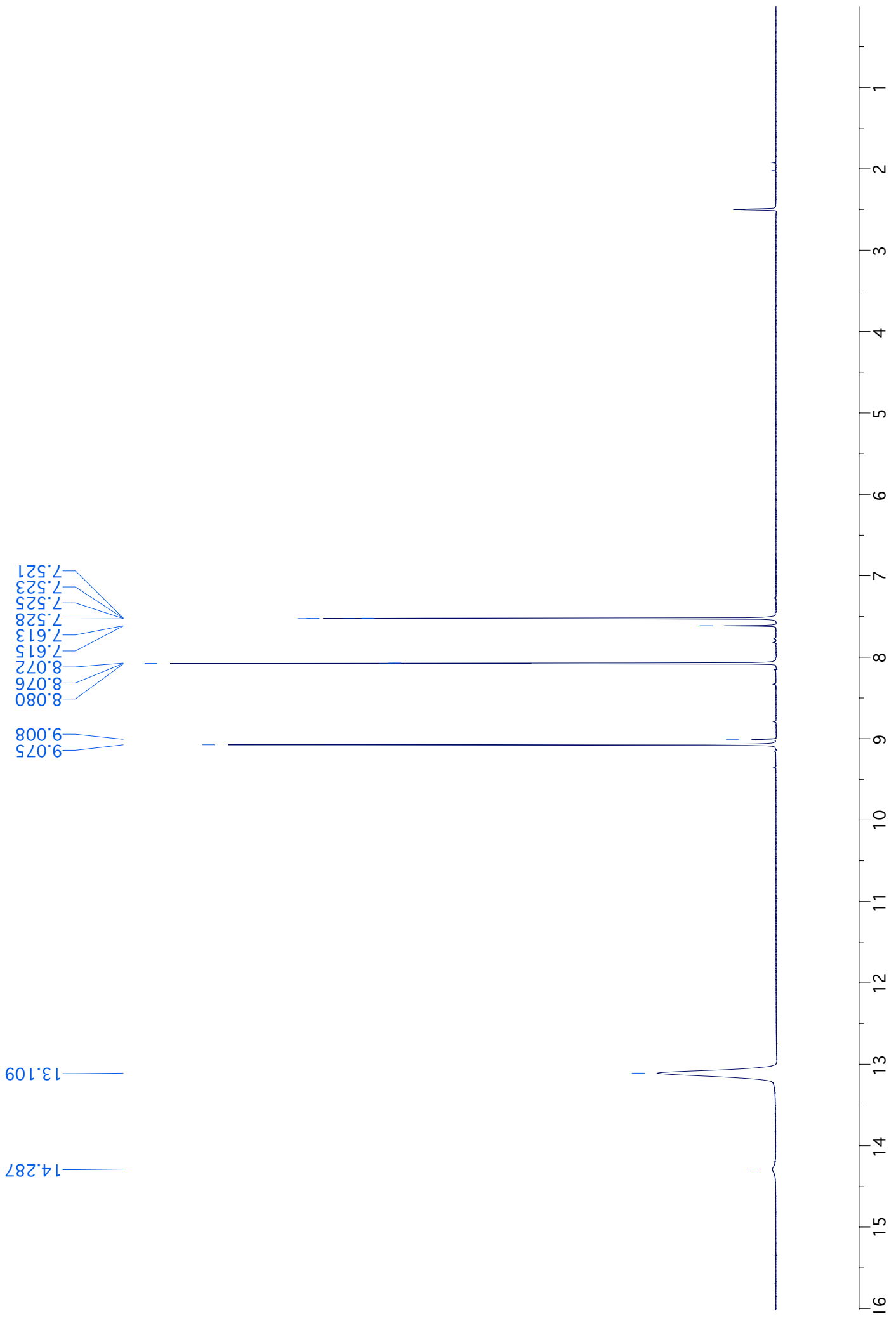


1- $\mathrm{H}_{2} \mathrm{SO}_{4}{ }^{1} \mathrm{HNMR}$ (Method B product)

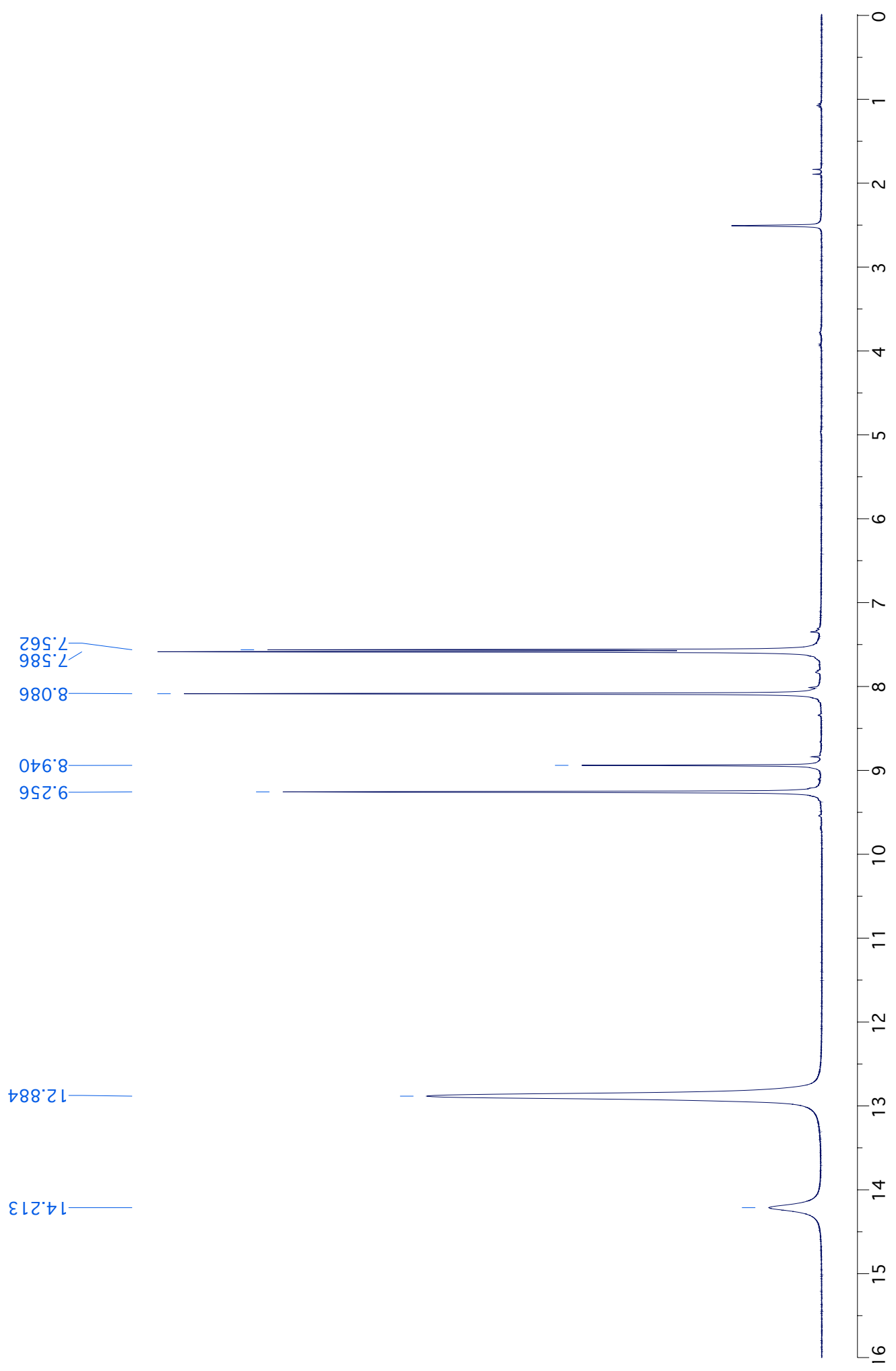


1-H $\mathrm{HO}_{4}{ }^{13} \mathrm{CNMR}$ (Method A product)

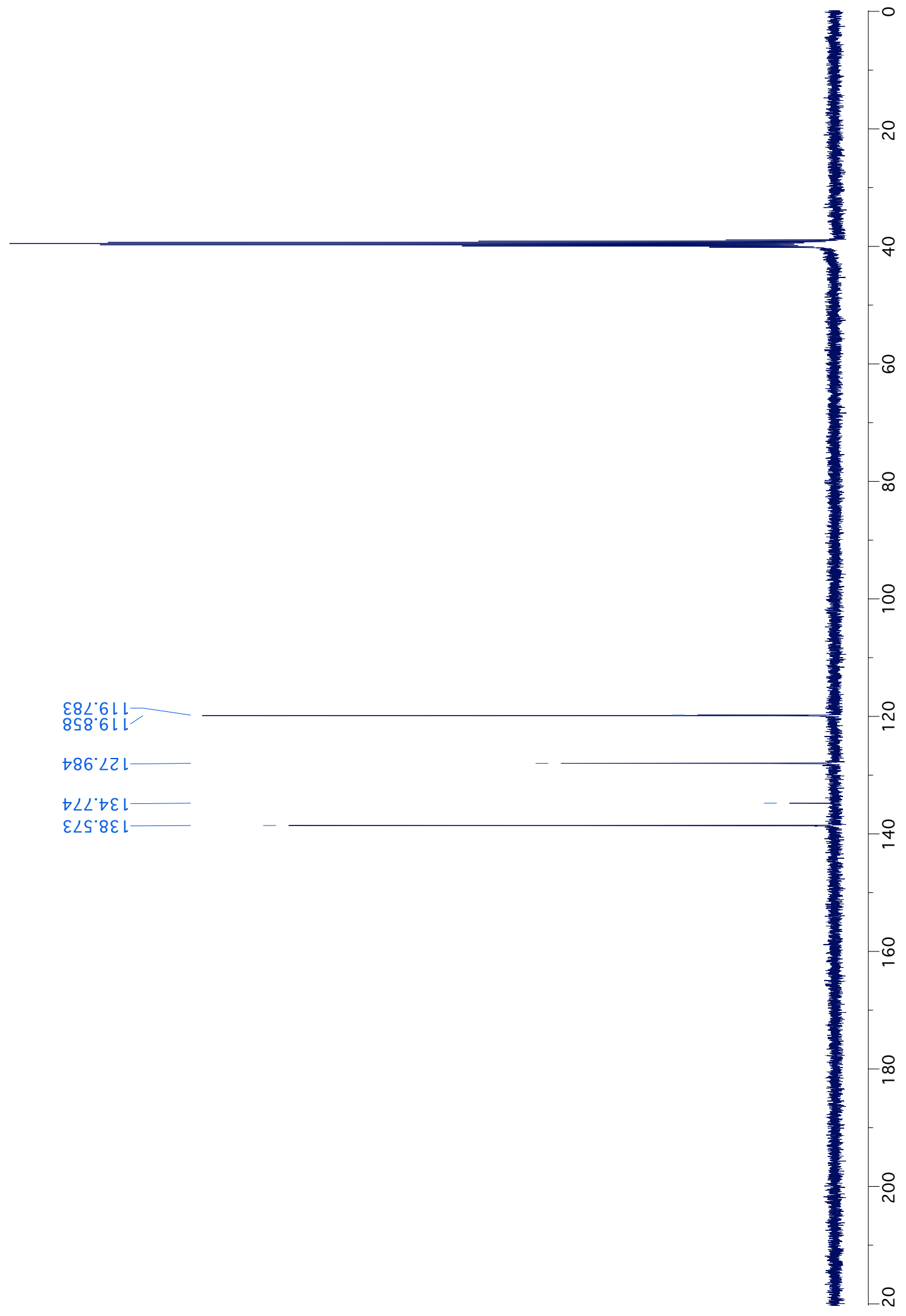


1-H $\mathrm{HO}_{4}{ }^{13} \mathrm{CNMR}$ (Method B product)

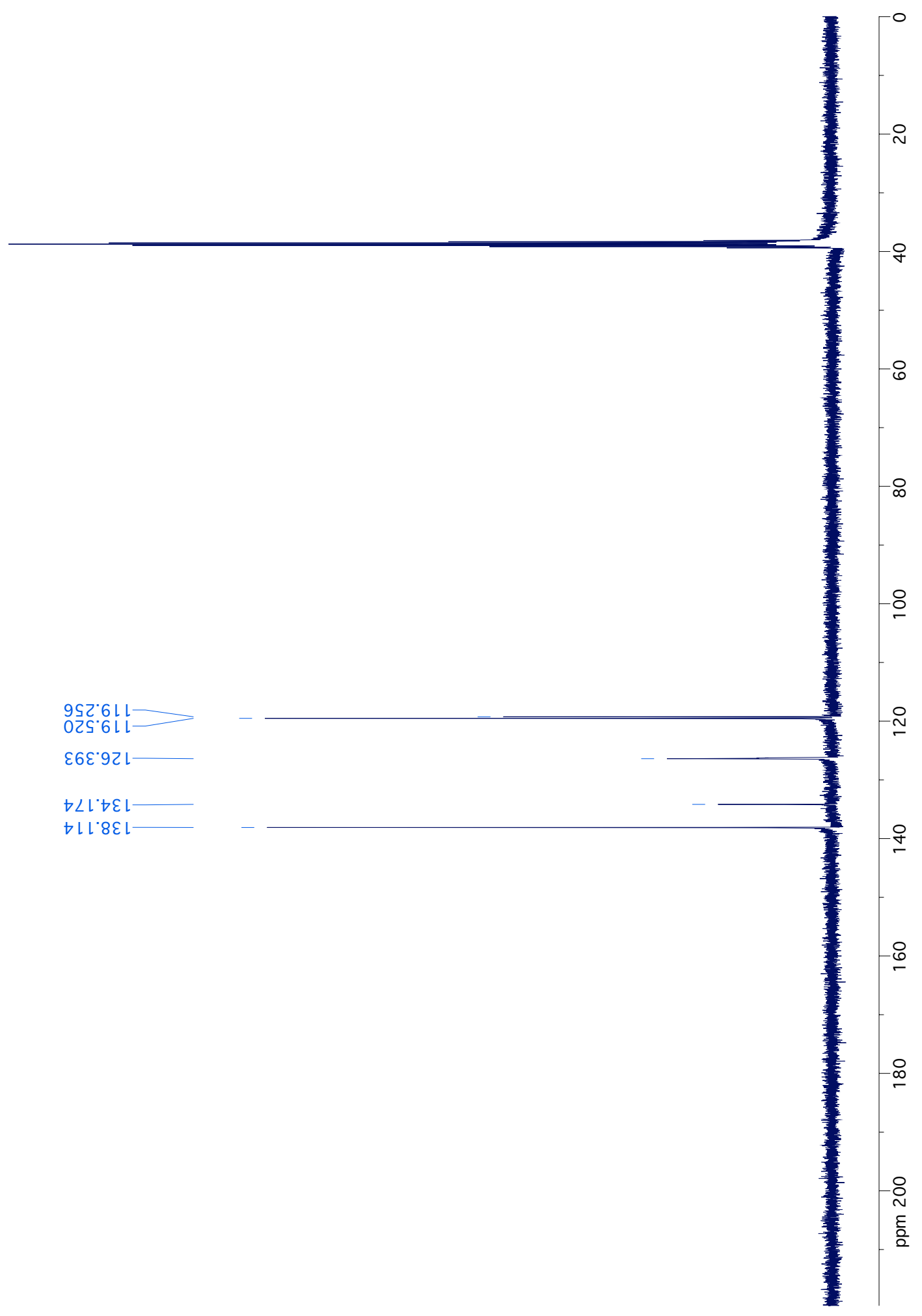


1- $\mathrm{H}_{2} \mathrm{SO}_{4}$ FTIR (Method A product)

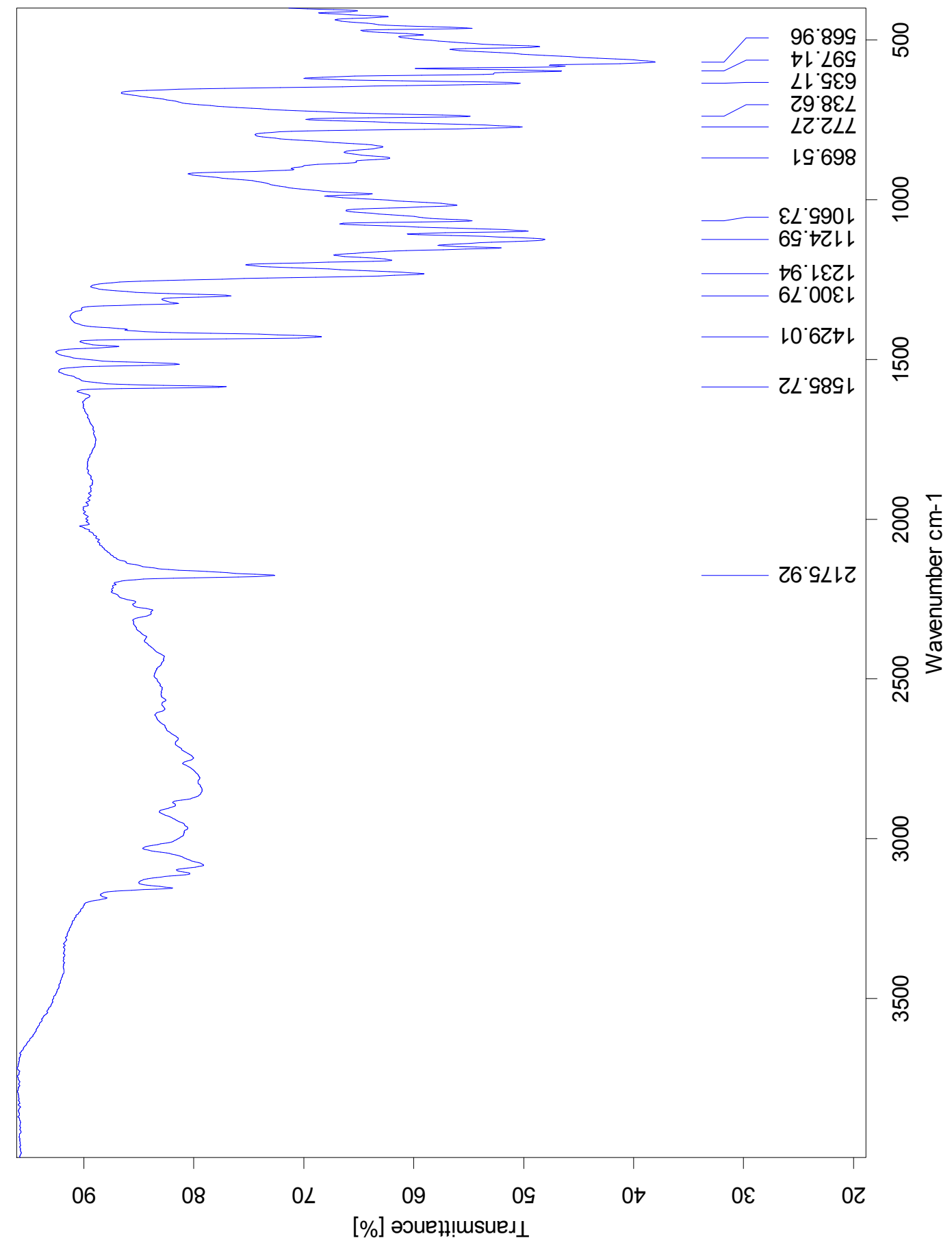


1- $\mathrm{H}_{2} \mathrm{SO}_{4}$ FTIR (Method B product)

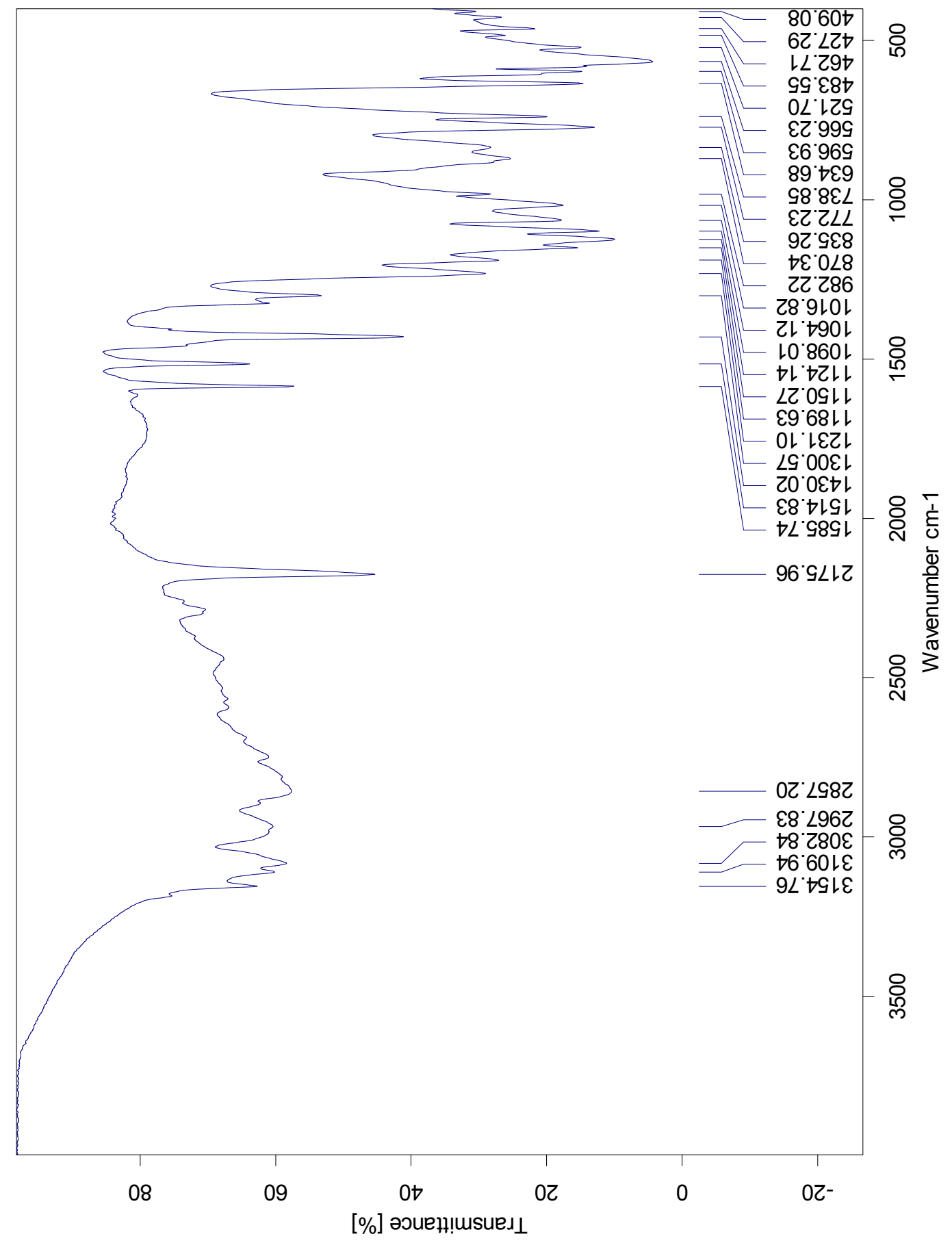




\section{1- $\mathbf{H}_{2} \mathrm{SO}_{4}$ MALDI in CHCA MeOH/DMSO (Method A product)}

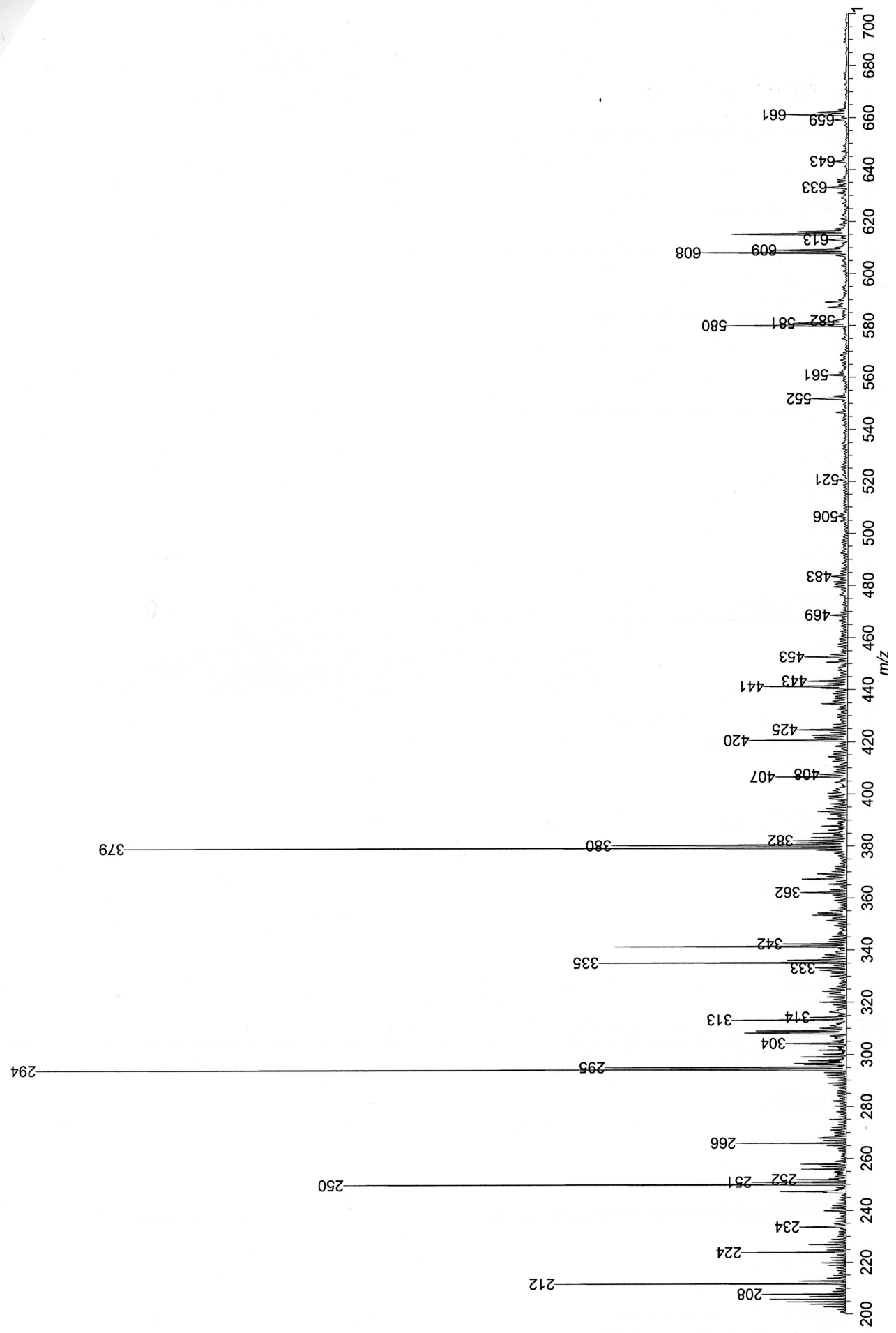


$\mathbf{1}-\mathrm{H}_{2} \mathrm{SO}_{4}$ MALDI in CHCA MeOH/DMSO (Method A product)

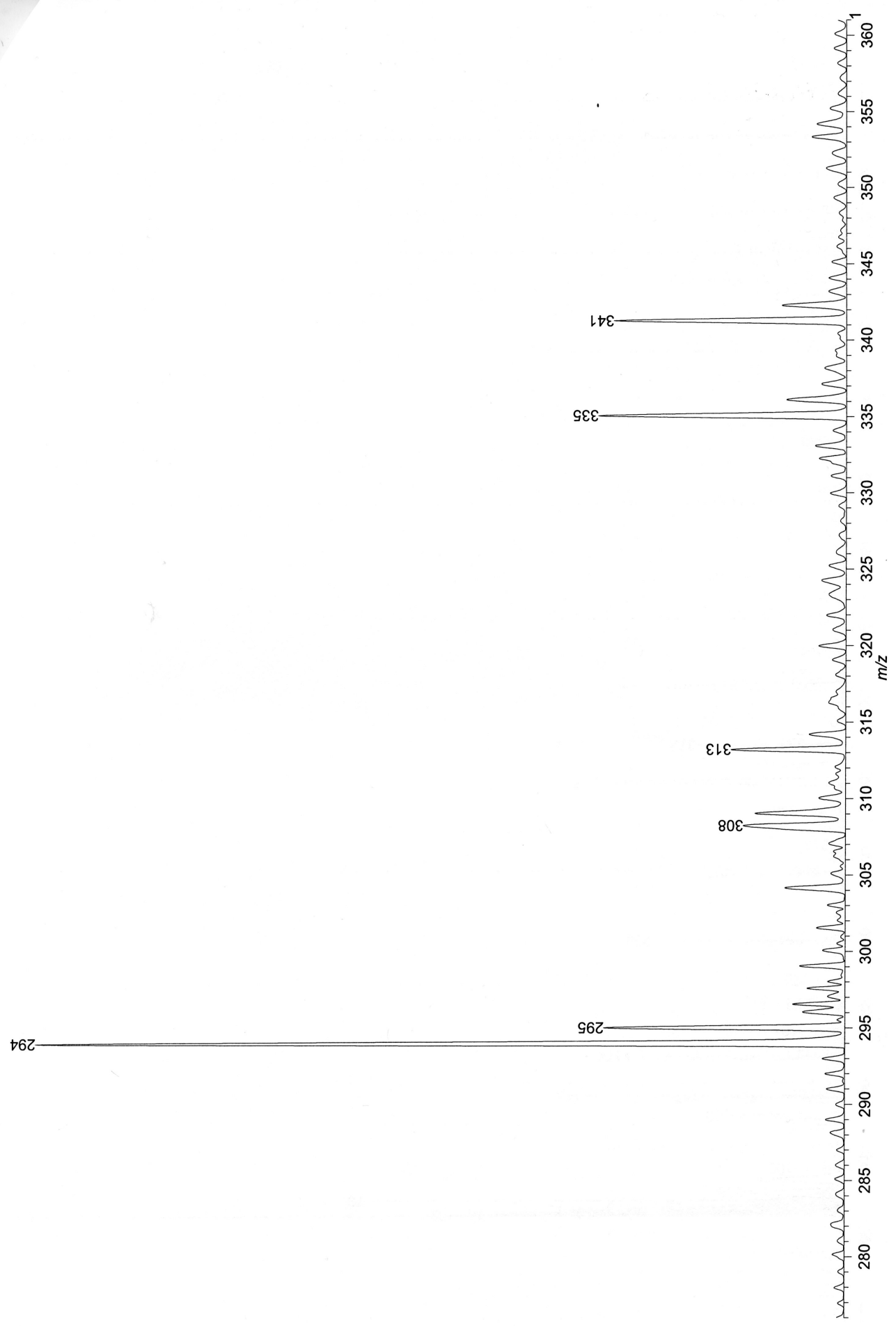


1- $\mathrm{H}_{2} \mathrm{SO}_{4}$ MALDI in CHCA MeOH/DMSO (Method A product)

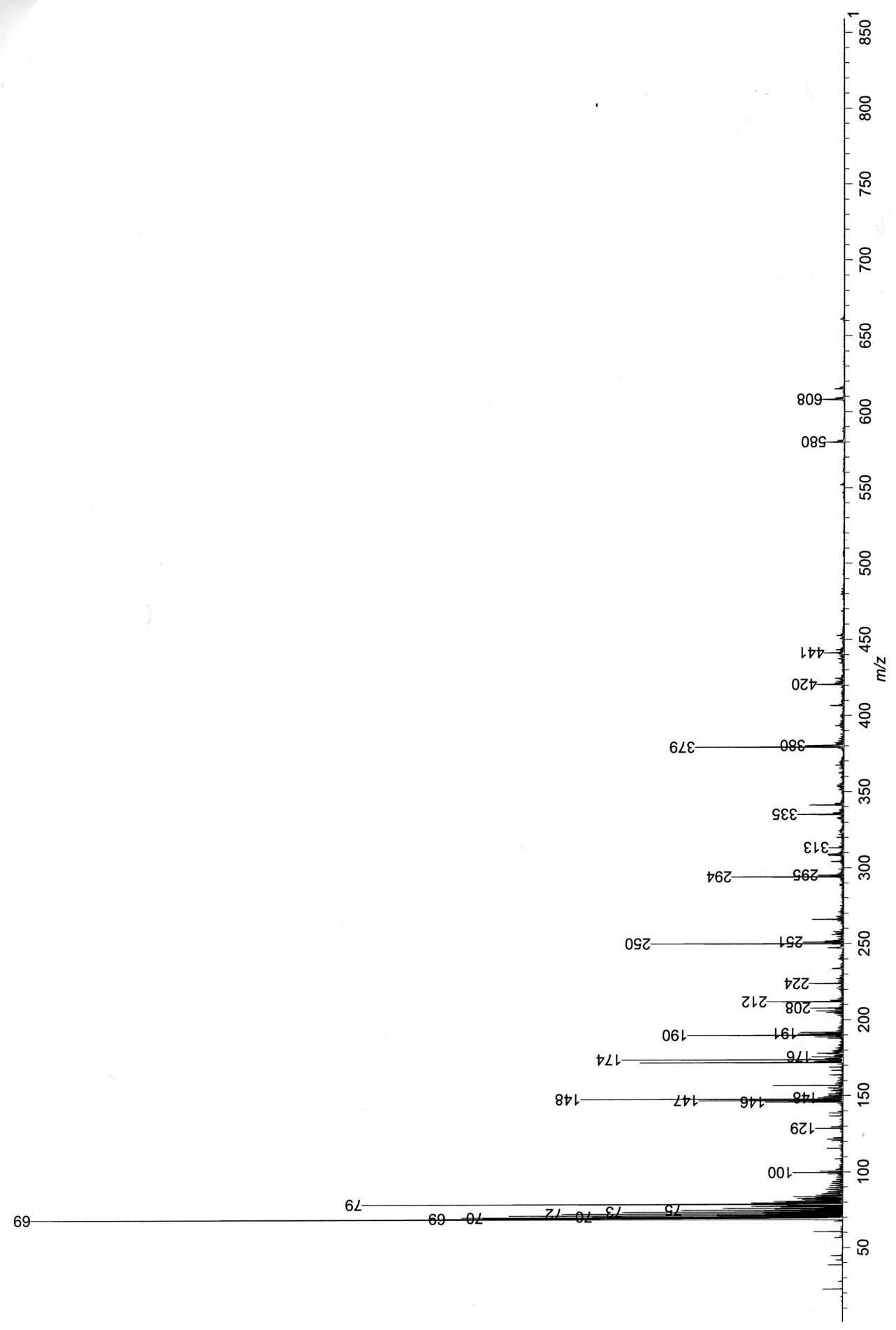


1- $\mathrm{H}_{2} \mathrm{SO}_{4}$ MALDI in CHCA ACN (Method B product)

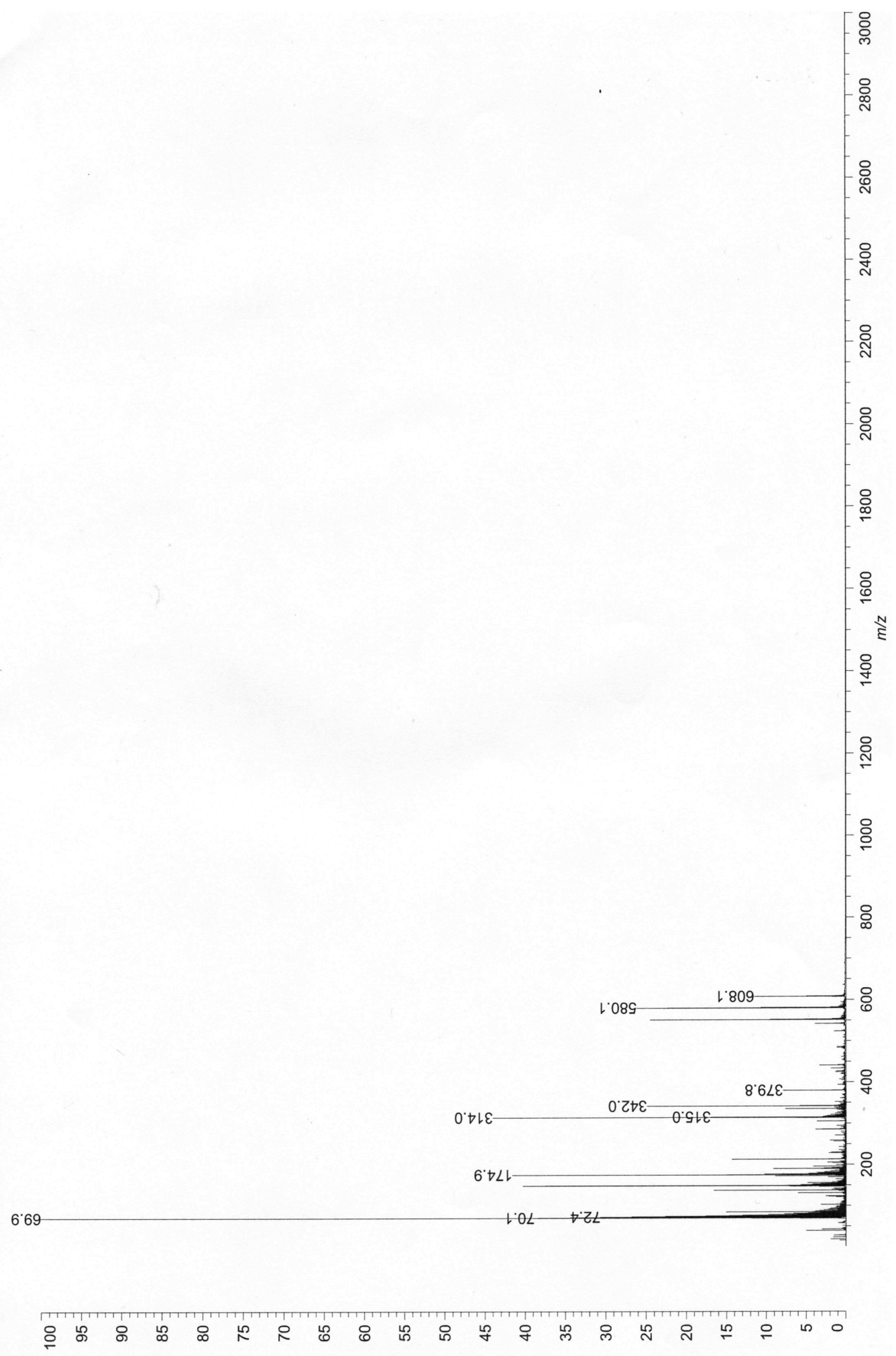


1- $\mathbf{H}_{2} \mathrm{SO}_{4}$ MALDI in CHCA ACN (Method B product)
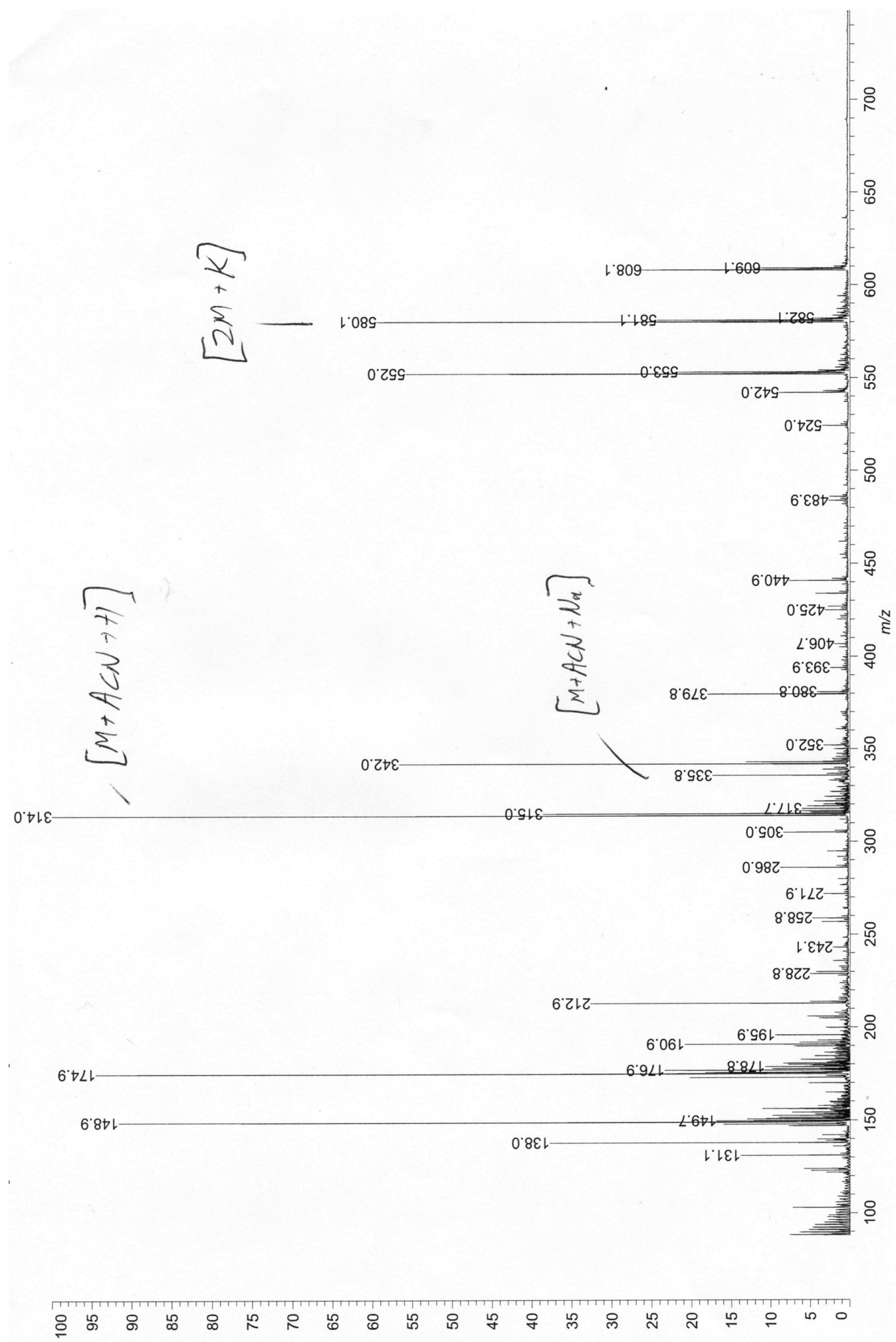
Photographic Stepwise Procedure (Method B)

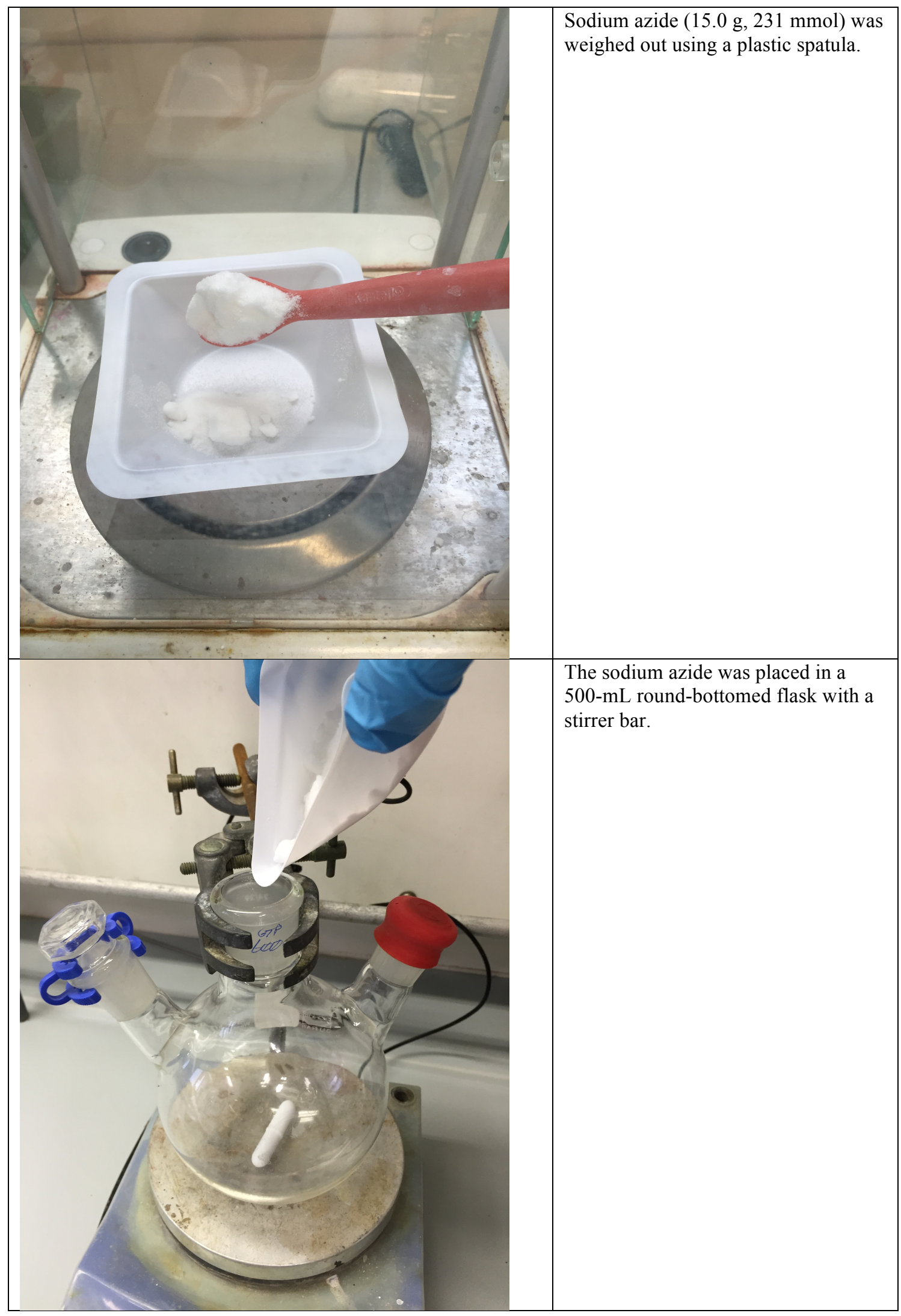


Photographic Stepwise Procedure (Method B)

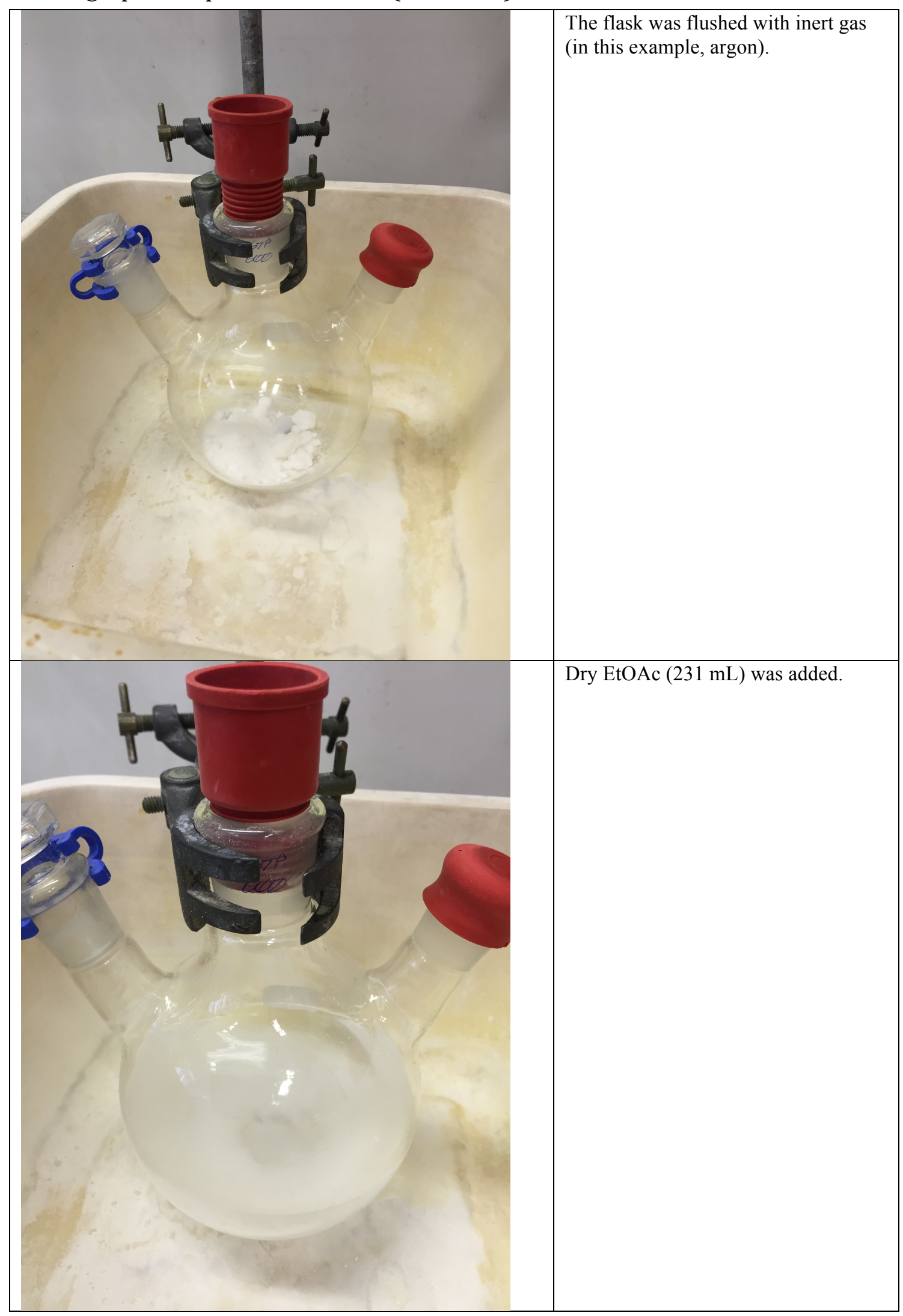


Photographic Stepwise Procedure (Method B)

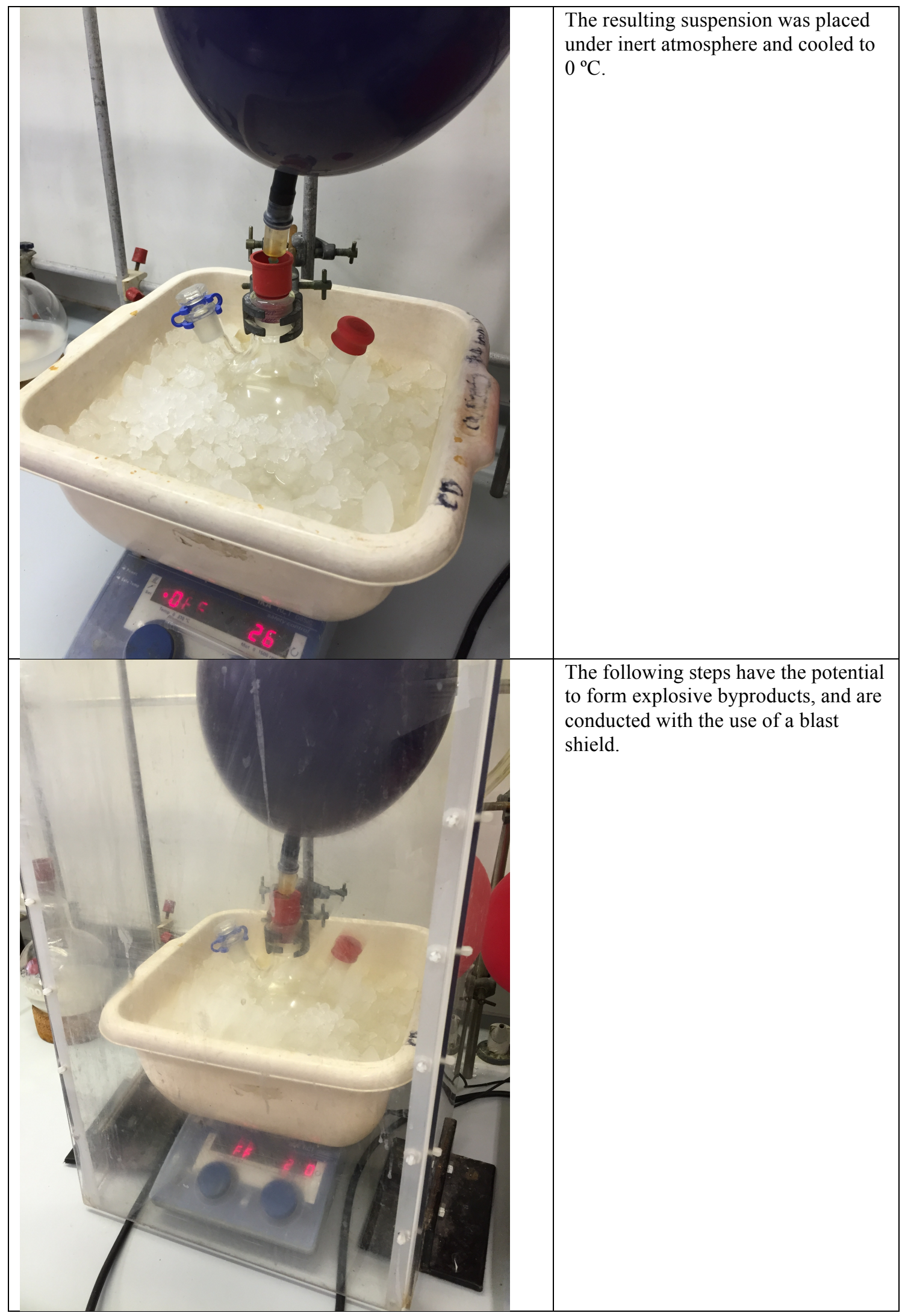


Photographic Stepwise Procedure (Method B)

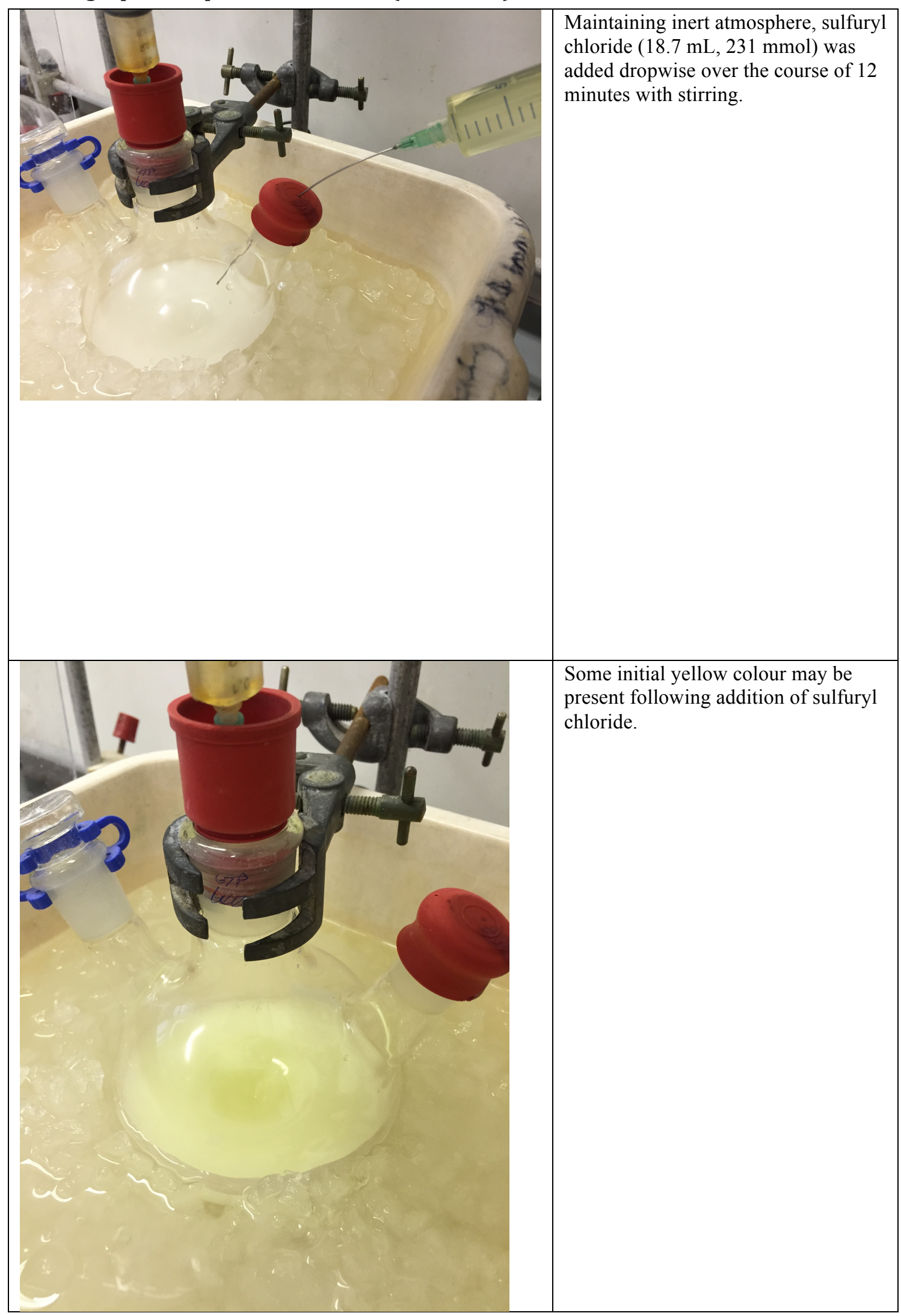


Photographic Stepwise Procedure (Method B)

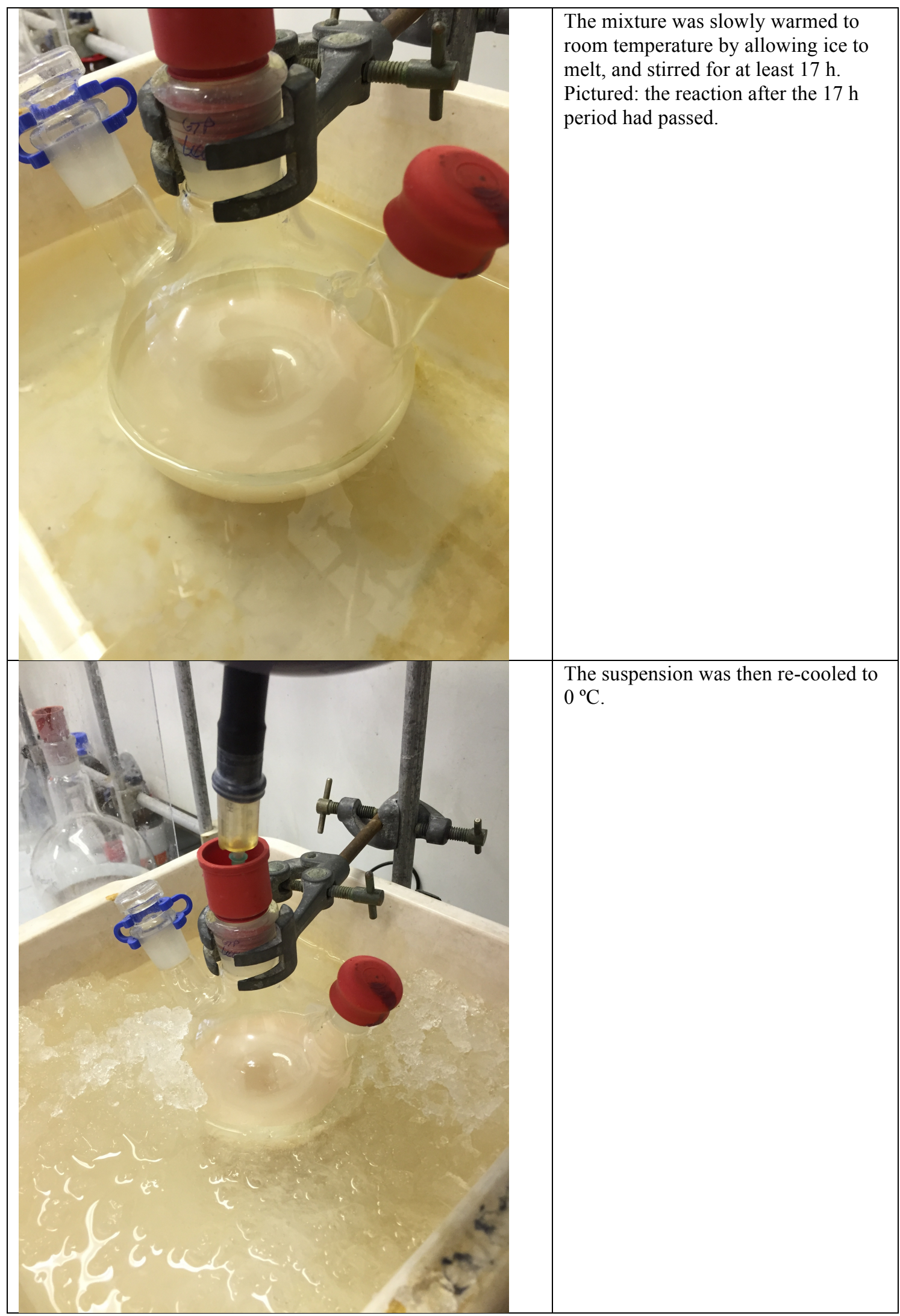


Photographic Stepwise Procedure (Method B)

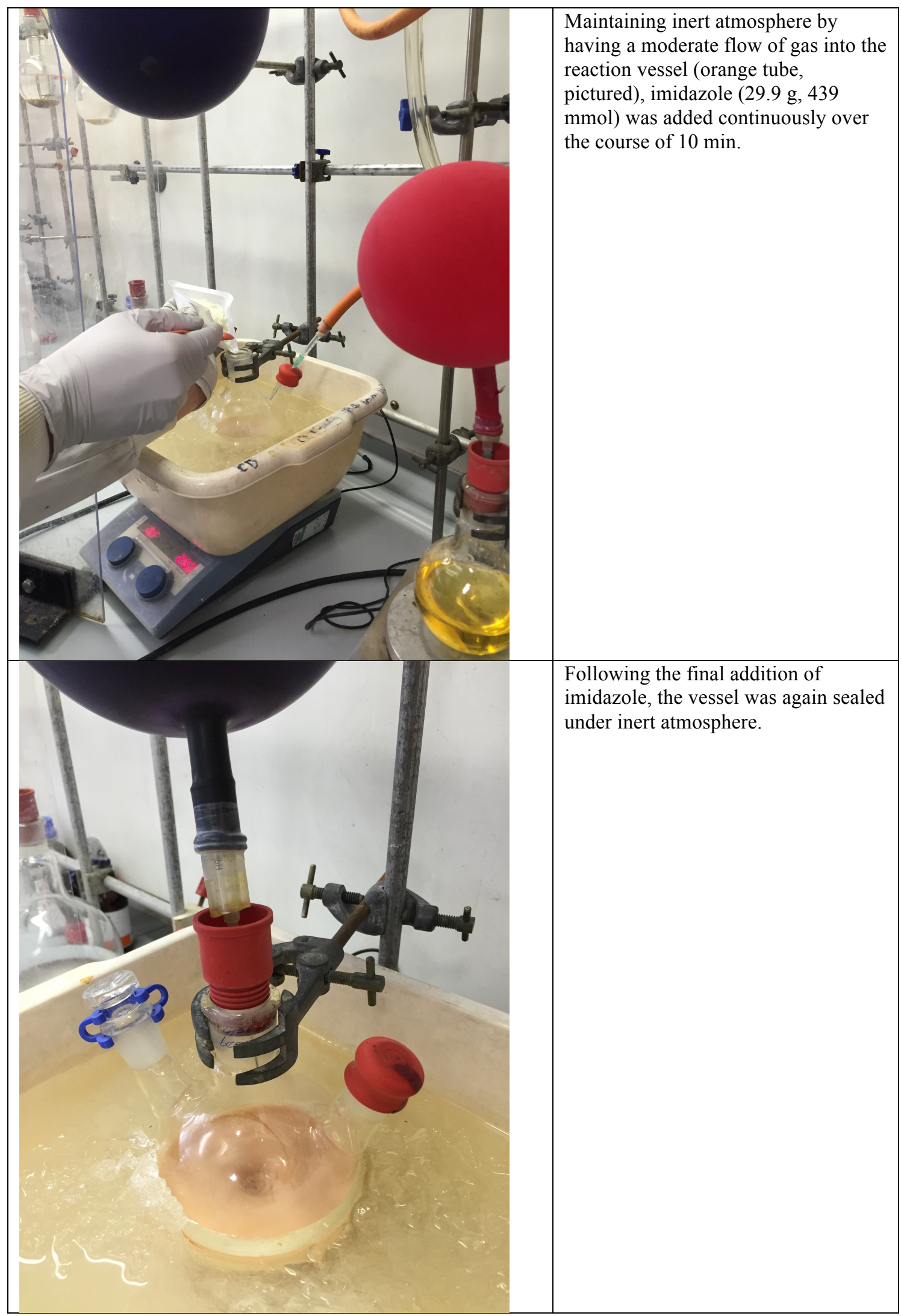


Photographic Stepwise Procedure (Method B)

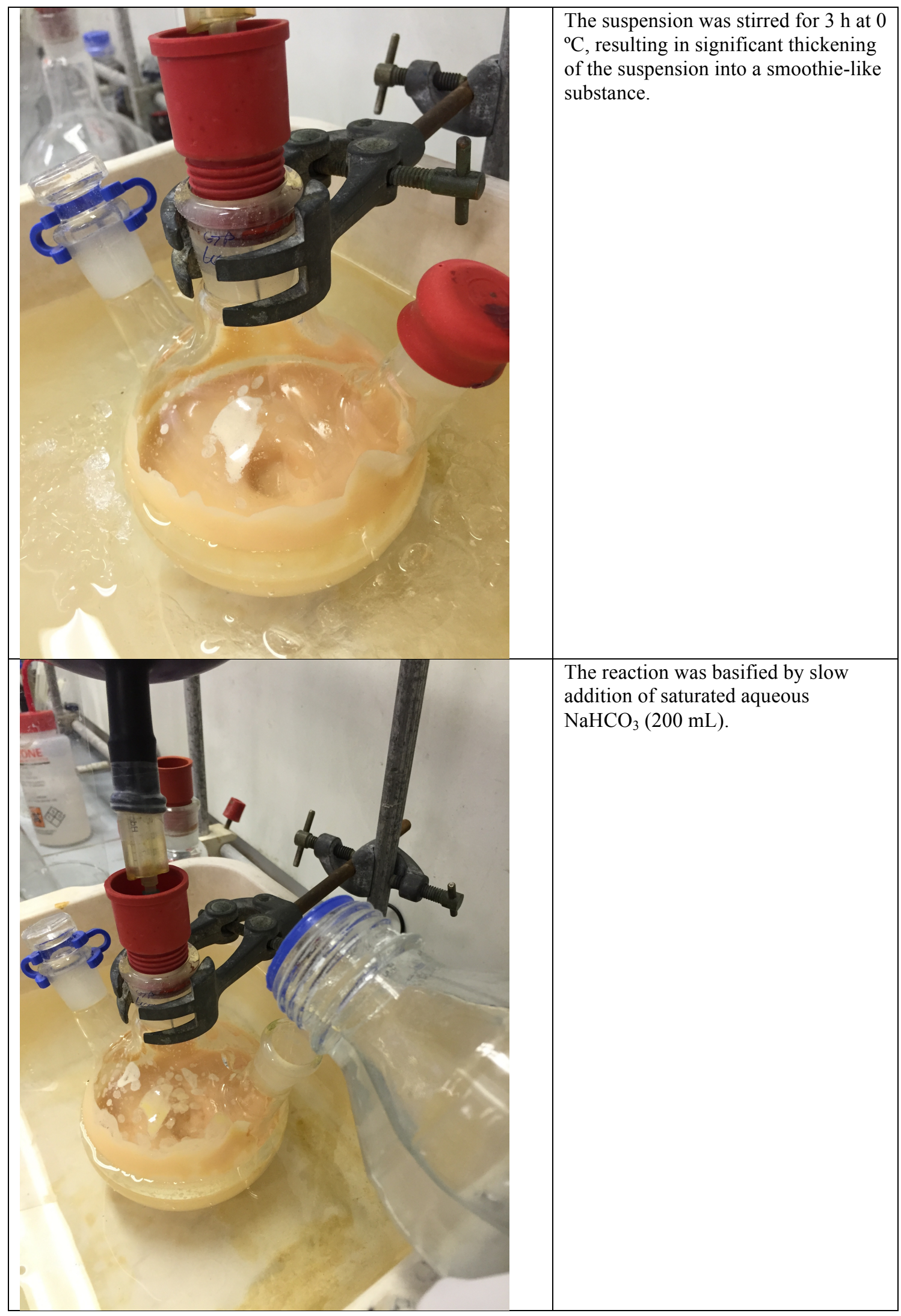


Photographic Stepwise Procedure (Method B)

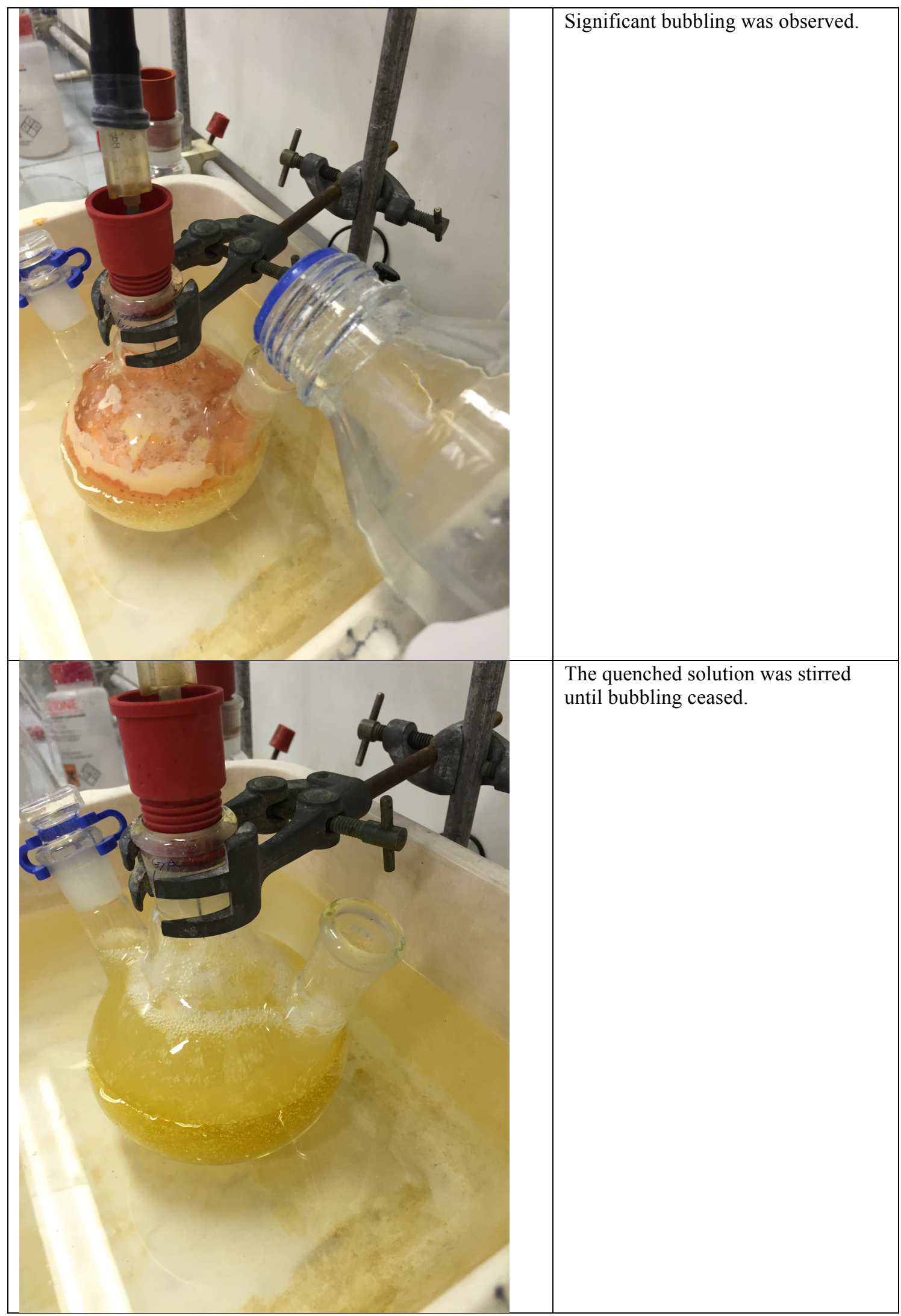


Photographic Stepwise Procedure (Method B)

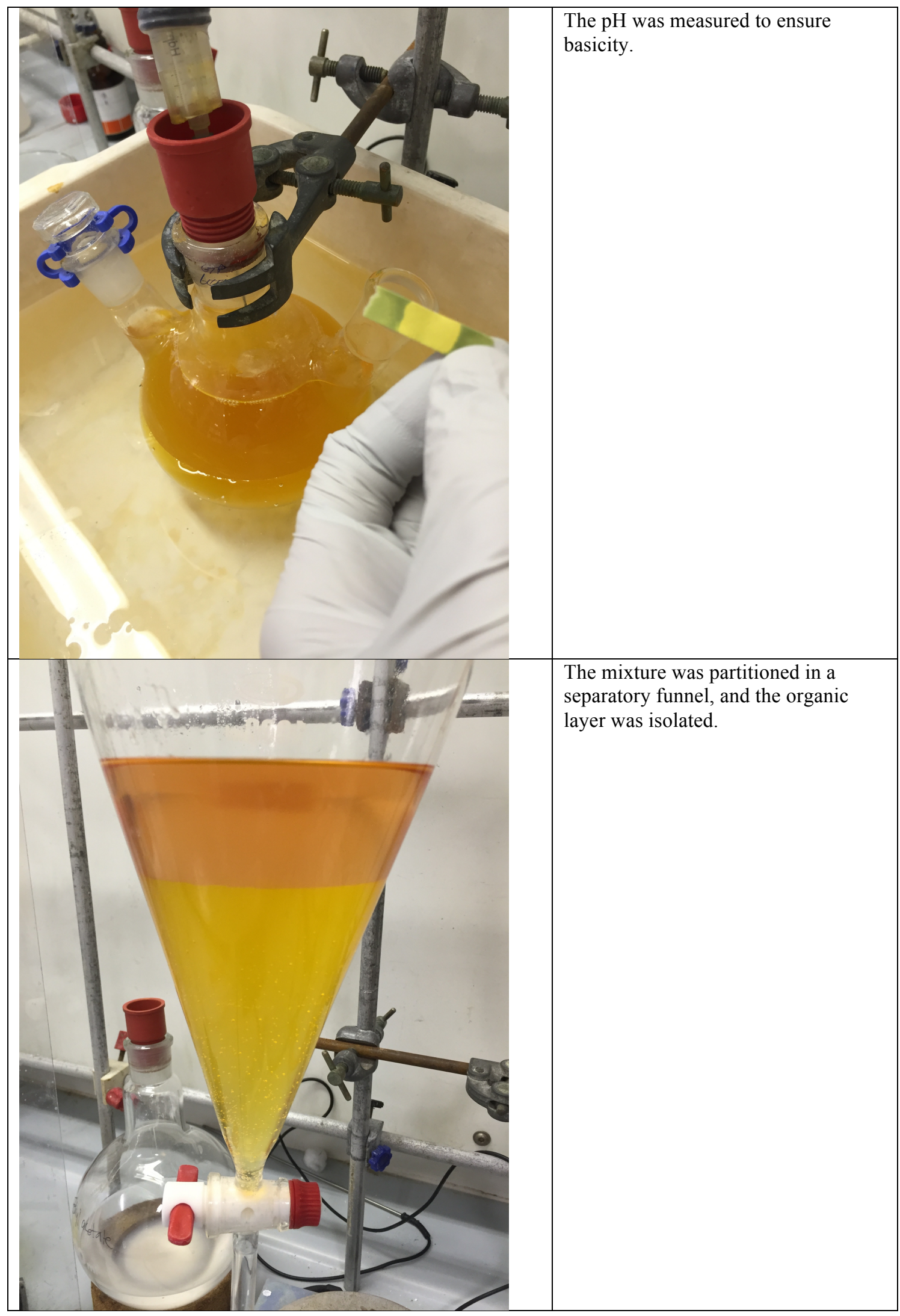


Photographic Stepwise Procedure (Method B)

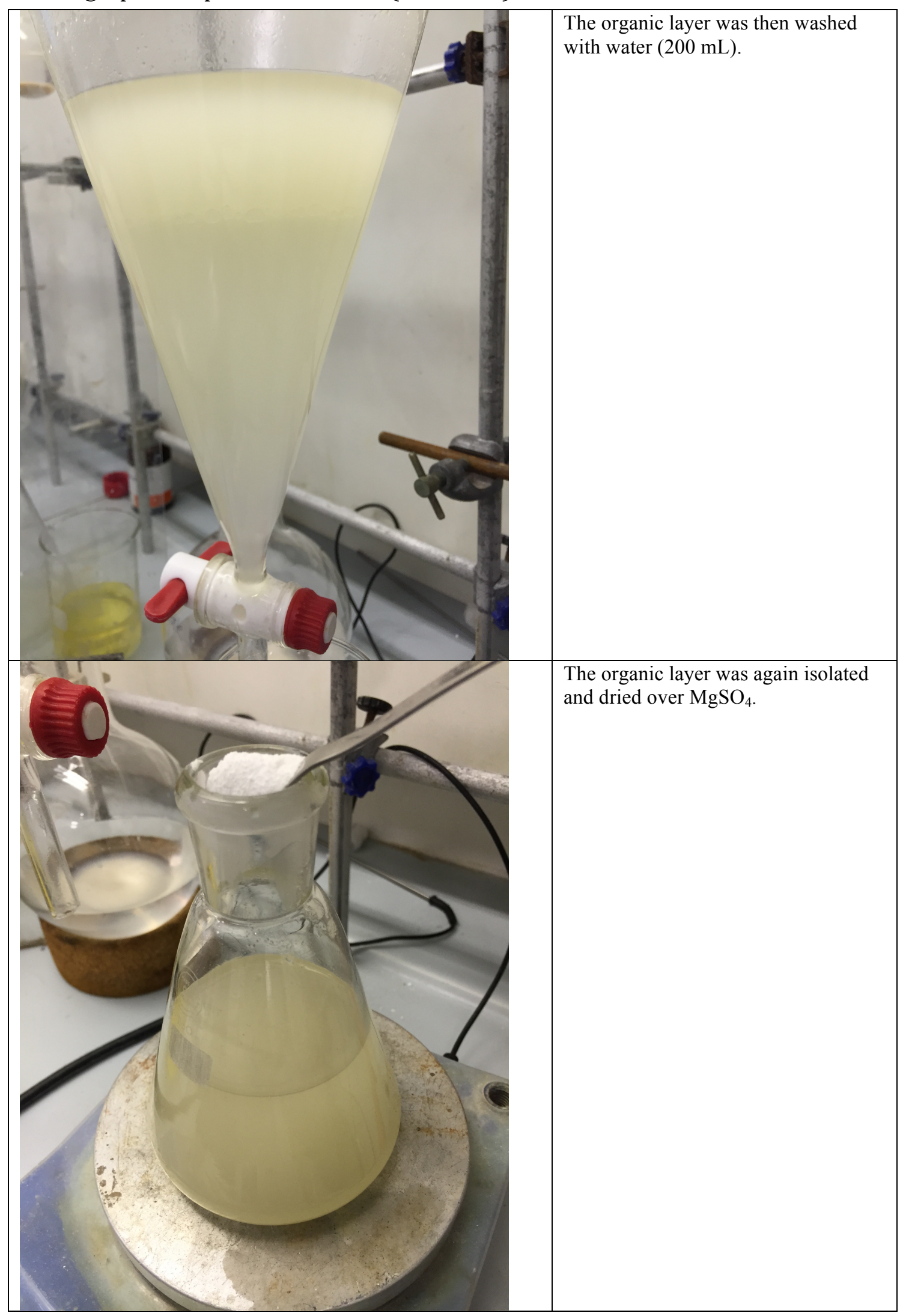


Photographic Stepwise Procedure (Method B)

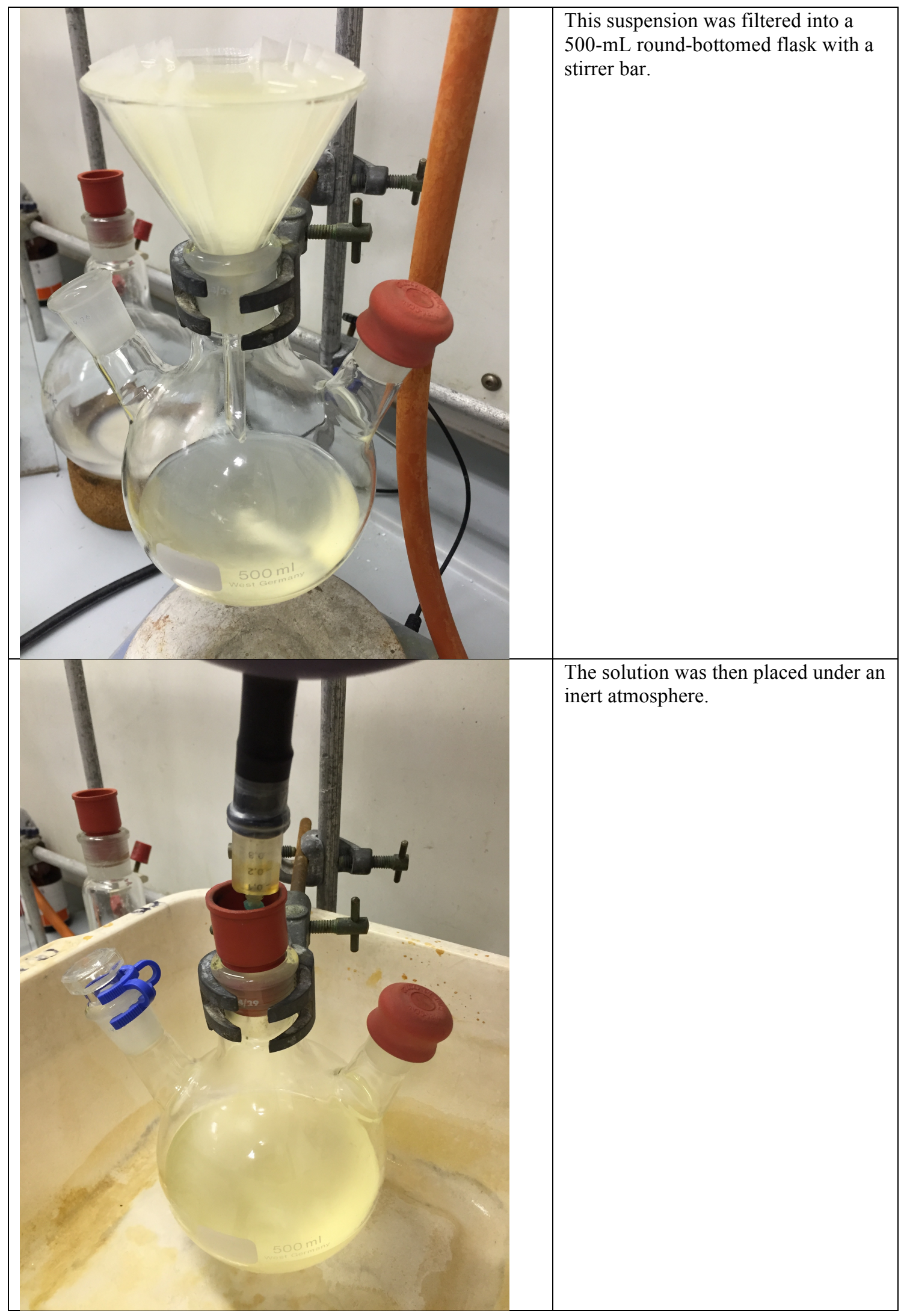


Photographic Stepwise Procedure (Method B)

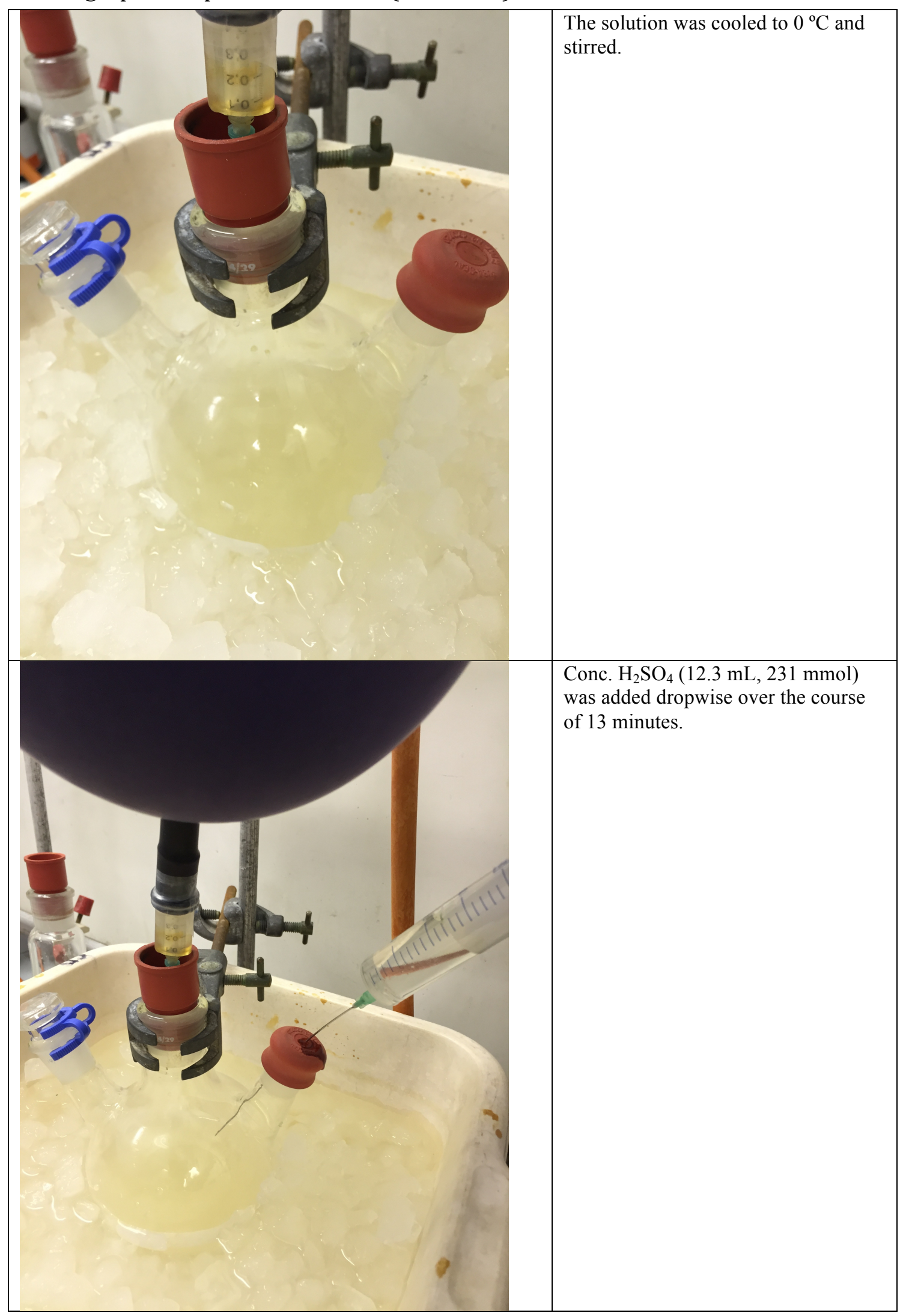


Photographic Stepwise Procedure (Method B)

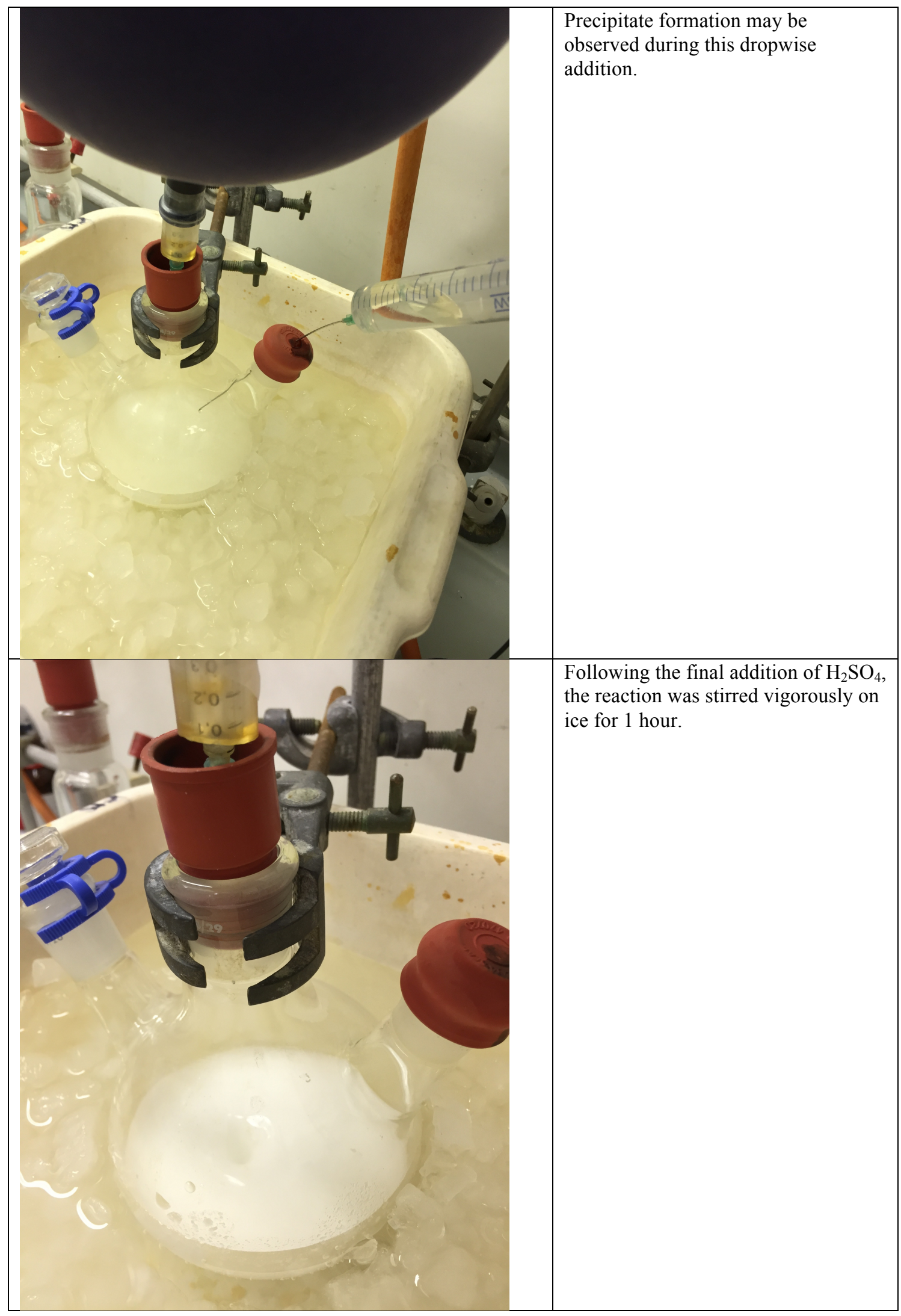


Photographic Stepwise Procedure (Method B)

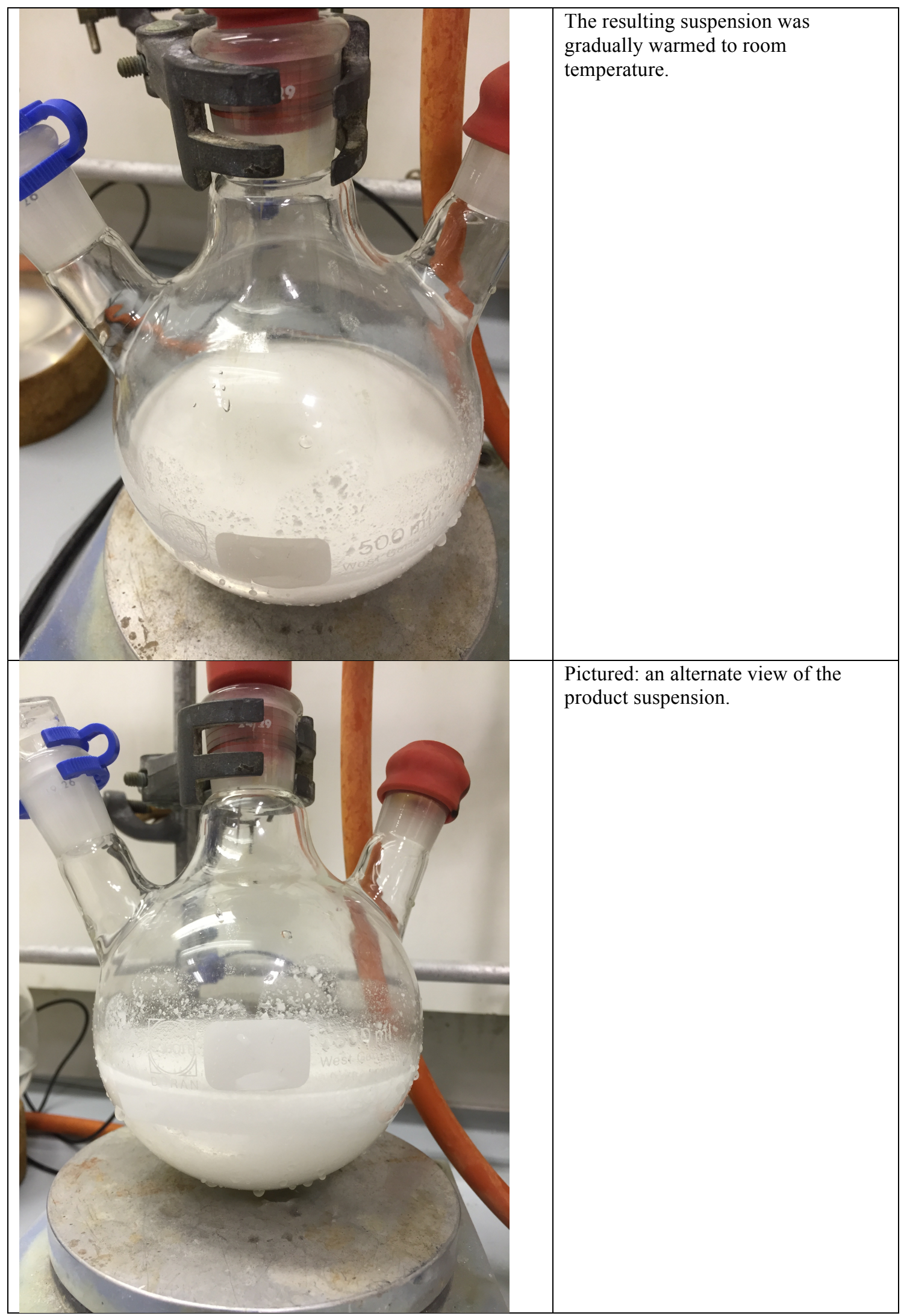


Photographic Stepwise Procedure (Method B)

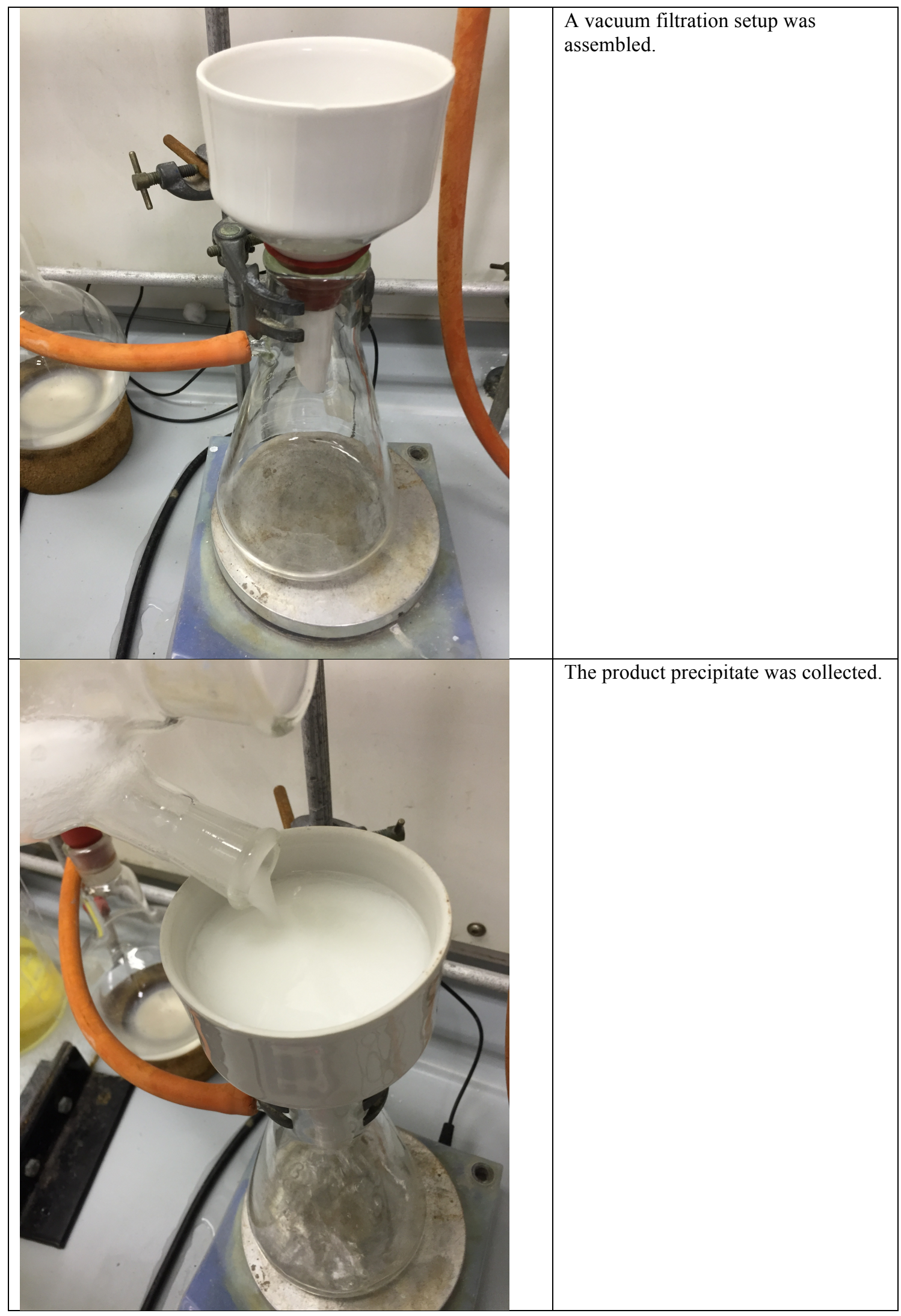


Photographic Stepwise Procedure (Method B)

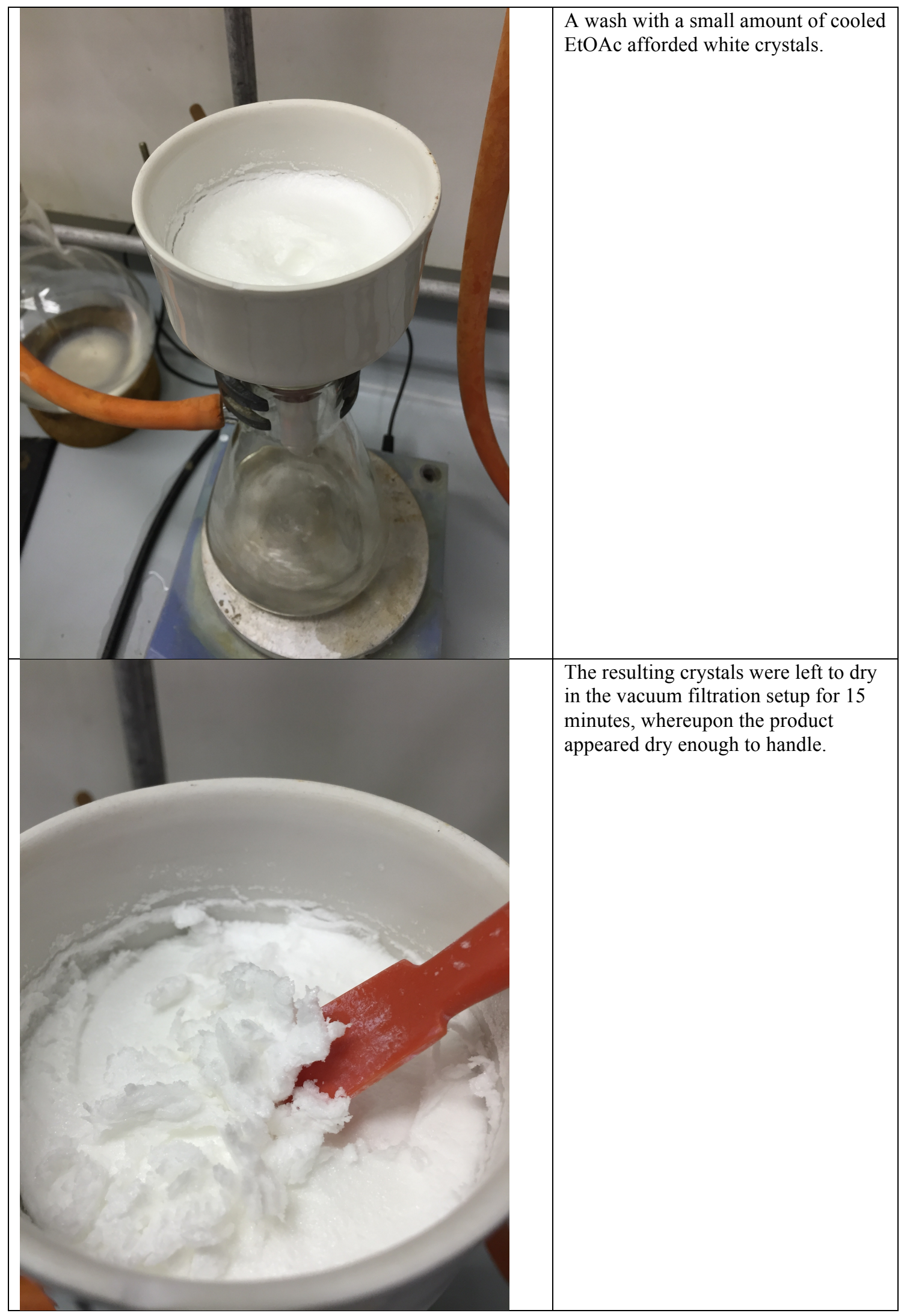


Photographic Stepwise Procedure (Method B)

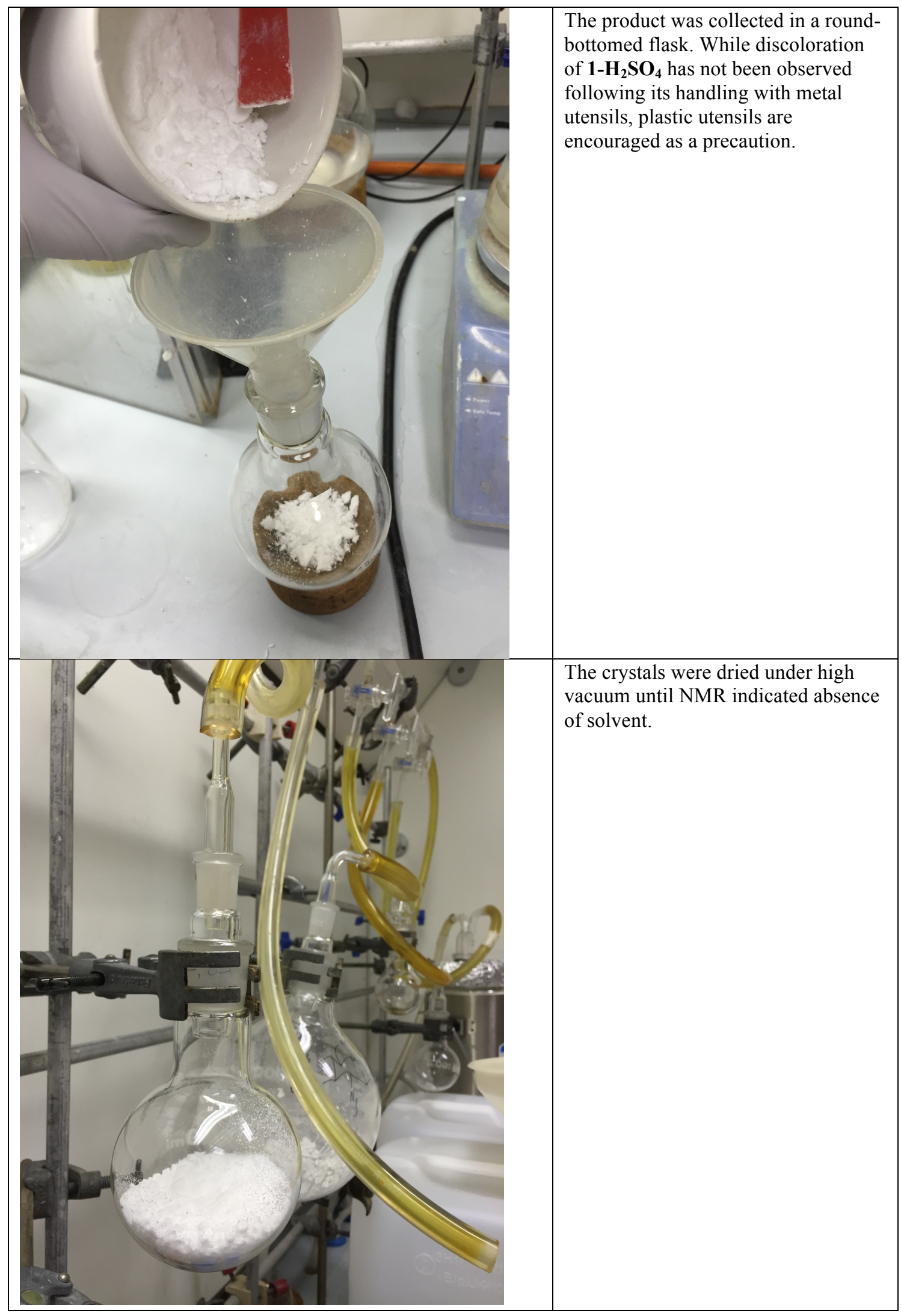


Photographic Stepwise Procedure (Method B)

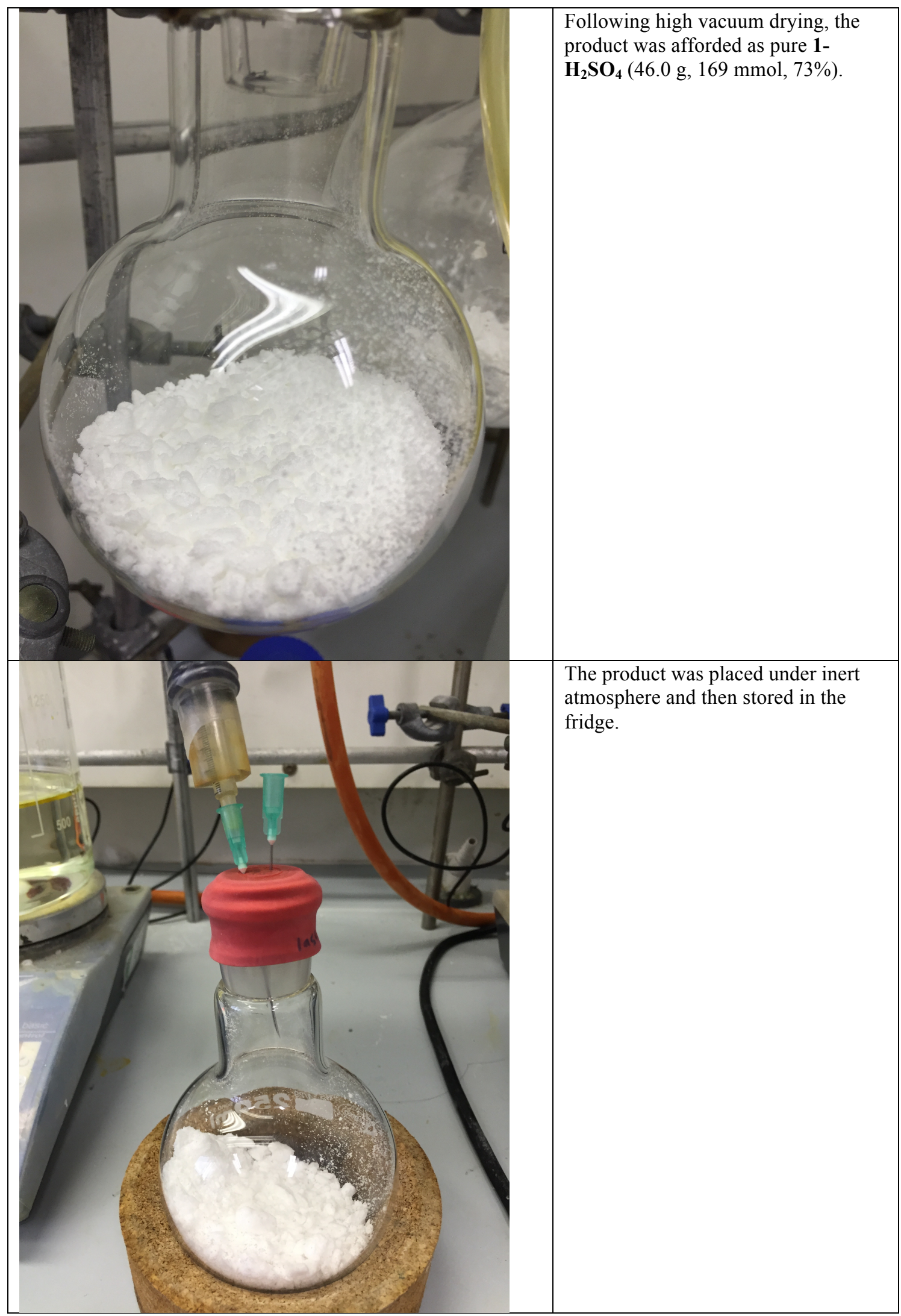


Photographic Stepwise Procedure (Method B)

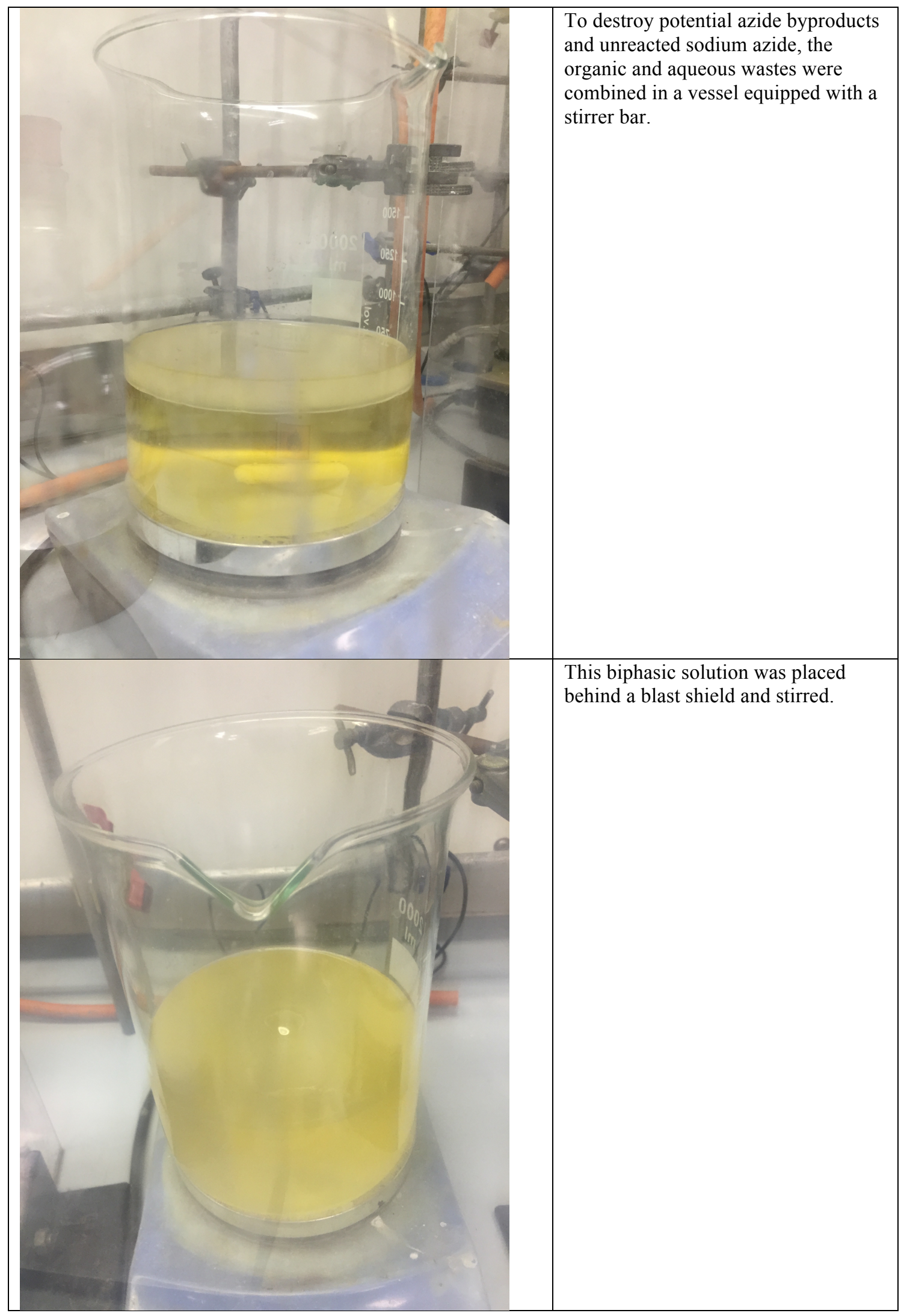


Photographic Stepwise Procedure (Method B)

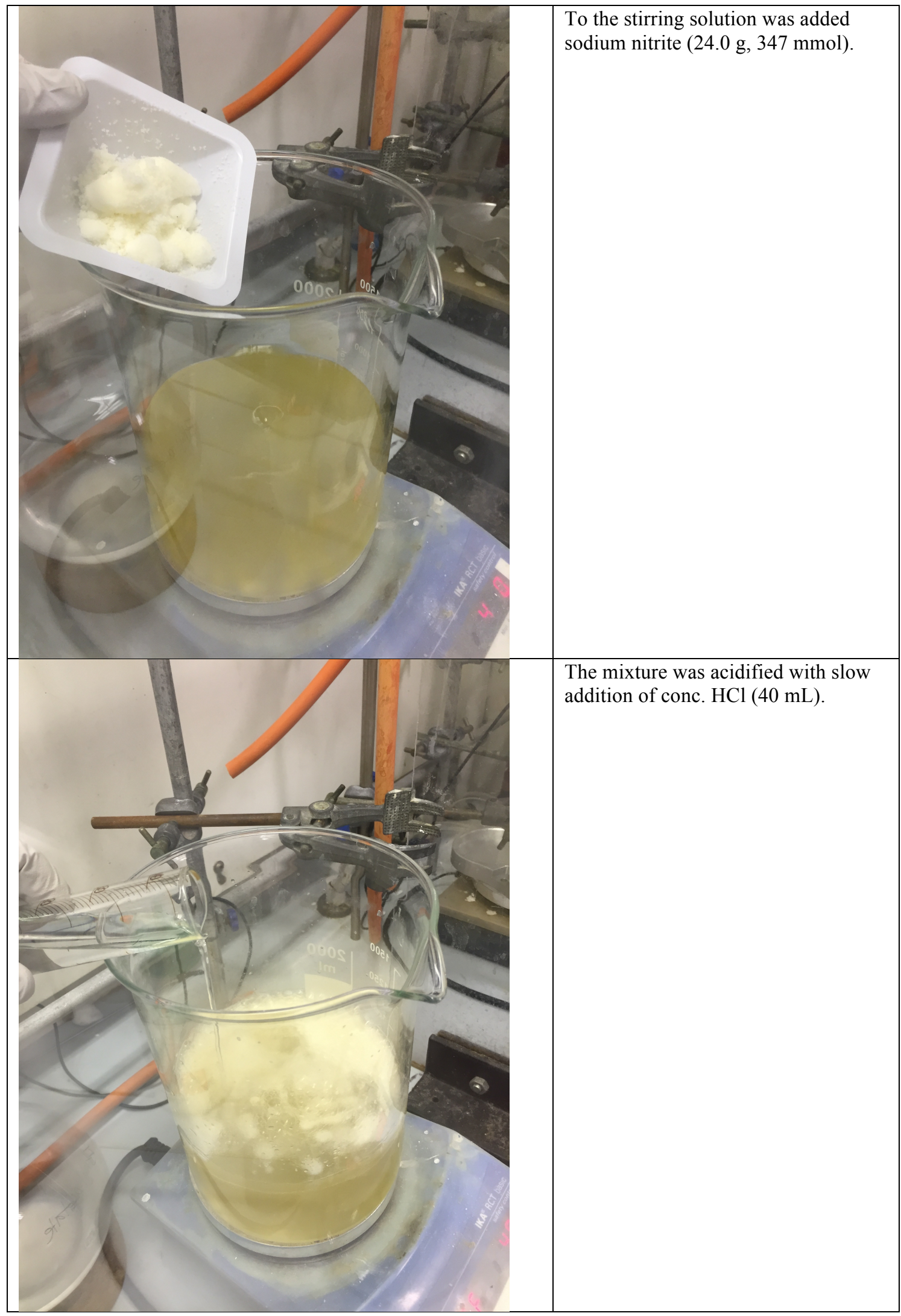


Photographic Stepwise Procedure (Method B)

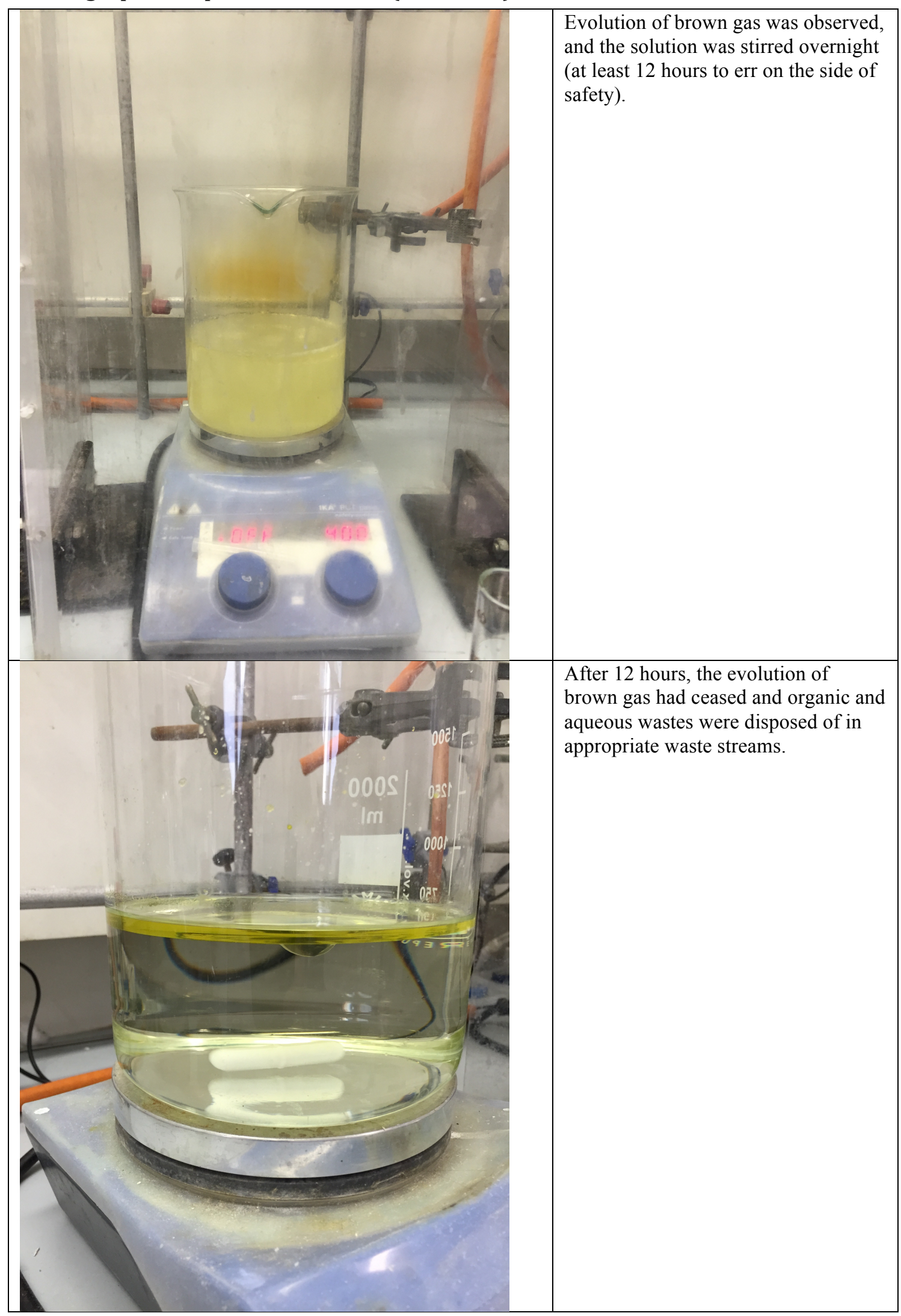




\section{Representative Diazotransfer Protocol}

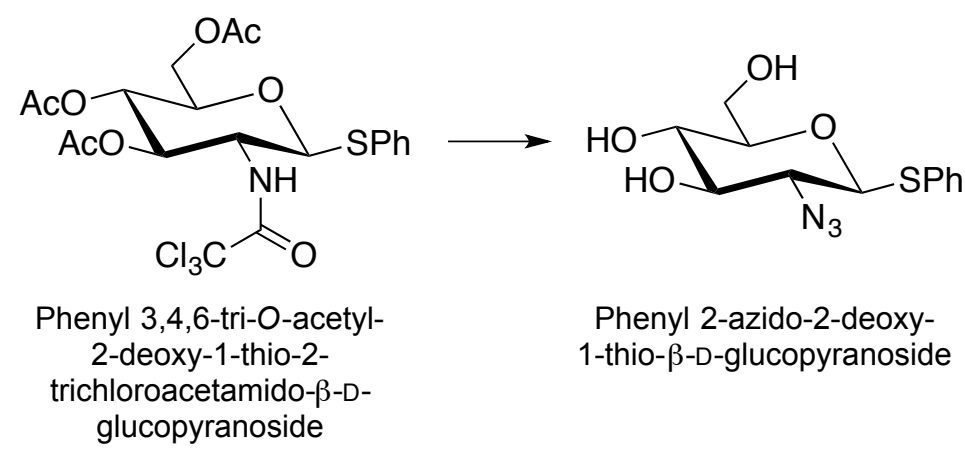

Phenyl 3,4,6-tri- $\boldsymbol{O}$-acetyl-2-deoxy-1-thio-2-trichloroacetamido-p-D-glucopyranoside $\quad\left(\begin{array}{lll}33.4 & \mathrm{~g}, & 61.5\end{array}\right.$ mmol) was suspended in $\mathrm{MeOH}(300 \mathrm{~mL})$ and stirred at room temperature. $\mathrm{K}_{2} \mathrm{CO}_{3}(17.9 \mathrm{~g}, 129.2 \mathrm{mmol})$ was dissolved in $\mathrm{H}_{2} \mathrm{O}(60 \mathrm{~mL})$ and added to the stirring suspension. The reaction was heated to $60{ }^{\circ} \mathrm{C}$ for $17 \mathrm{~h}$ whereupon the reaction was judged to be complete by consumption of starting material by TLC (intermediate $\mathrm{Rf}=0.30,15 \% \mathrm{MeOH}$ in EtOAc). The suspension was cooled to room temperature and filtered through Celite ${ }^{\circledR}$. The resulting mother liquor was cooled to $0{ }^{\circ} \mathrm{C}$ and to the solution was added 1$\mathrm{H}_{2} \mathrm{SO}_{4}(17.9 \mathrm{~g}, 64.6 \mathrm{mmol}), \mathrm{Cu}(\mathrm{II}) \mathrm{SO}_{4}(0.15 \mathrm{~g}, 0.6 \mathrm{mmol})$, and $\mathrm{K}_{2} \mathrm{CO}_{3}(17.9 \mathrm{~g}, 129.2 \mathrm{mmol})$. The suspension was brought gradually to room temperature and stirred for 3.5 hours, whereupon the reaction was judged to be complete by consumption of intermediate by TLC (product $\mathrm{Rf}=0.61,100 \%$ EtOAc). The resulting milky white suspension was filtered, and the light green mother liquor was concentrated to remove $\mathrm{MeOH}$. The resulting aqueous solution was stirred vigorously and acidified to $\mathrm{pH}=2$ with the addition of $1 \mathrm{M} \mathrm{HCl}(\mathrm{aq})$. The aqueous solution was extracted with EtOAc $(3 \times 200 \mathrm{~mL})$. The organic layers were combined and washed with saturated aqueous $\mathrm{NaHCO}_{3}(2 \times 200 \mathrm{~mL})$ and brine $(2 \times 200 \mathrm{~mL})$. The solution was dried over $\mathrm{MgSO}_{4}$, filtered, then solvents removed in vacuo to afford Phenyl 2-azido-2deoxy-1-thio- $\beta$-D-glucopyranoside as an off-white powder (16.5 g, $55.0 \mathrm{mmol}, 89 \%)$.

${ }^{1} \mathrm{H}-\mathrm{NMR}\left(\mathrm{CD}_{3} \mathrm{OD}, 400 \mathrm{MHz}\right) \delta$ 7.62-7.59 (m, $\left.2 \mathrm{H}, \mathrm{Ar}-\mathrm{H}\right), 7.38-7.32(\mathrm{~m}, 3 \mathrm{H}, \mathrm{Ar}-\mathrm{H}), 4.57$ (d, J = $10.4 \mathrm{~Hz}$, 1 H, H1), 3.91-3.87 (m, 1 H, H6a), 3.73-3.68 (m, 1 H, H6b), 3.45-3.41 (m, 1 H, H3), 3.33-3.31 (m, 2 H, H4 \& H5), $3.16(\mathrm{dd}, \mathrm{J}=10.0,9.6 \mathrm{~Hz}, 1 \mathrm{H}, \mathrm{H} 2) ;{ }^{13} \mathrm{C}-\mathrm{NMR}\left(\mathrm{CD}_{3} \mathrm{OD}, 100 \mathrm{MHz}\right) \delta 133.8(2 \mathrm{x} \mathrm{Ar}-\mathrm{H}), 133.6(\mathrm{Ar})$, 130.1 (2x Ar-H), 129.1 (Ar-H), 87.3 (C1), 82.2 (C5), 78.5 (C3), 71.2 (C4), 67.1 (C2), 56.5 (C6); ES MS $m / z 320\left(\mathrm{MNa}^{+}, 100 \%\right)$; HRMS Calculated for $\mathrm{C}_{12} \mathrm{H}_{15} \mathrm{~N}_{3} \mathrm{O}_{4} \mathrm{Na}^{+}: 320.0676$, Found: 320.0677; FTIR (neat) $v_{\max } 3257$ (s, br, OH stretch), 2111 (s, azide stretch), $\mathrm{cm}^{-1}$. The data obtained was consistent to that which was reported in the literature. ${ }^{1}$ Furthermore, azide conversion is comparable (literature procedure using 1HCl yielded $92 \%$ product). ${ }^{1}$

1. Hansen, S.; Miller, G.; Baráth, M.; Broberg, K.; Avizienyte, E.; Helliwell, M.; Raftery, J.; Jayson, G.; Gardiner, J. J. Org. Chem. 2012, 77, 7823, DOI: 10.1021/jo300722y 